\title{
Quasi-explicit time-integration schemes for dynamic fracture with set-valued cohesive zone models
}

\author{
D. Doyen · A. Ern · S. Piperno
}

Received: date / Accepted: date

\begin{abstract}
We investigate quasi-explicit time-integration schemes for solving dynamic fracture problems with set-valued cohesive zone models. These schemes combine a central difference time-integration scheme and a partially implicit and lumped treatment of the cohesive forces. At each time step, the displacements of the nodes in the interior of the domain are computed in an explicit way, while the displacements of each node at the interface are computed by solving a local nonlinear problem. The method provides a general and robust way of treating the set-valued cohesive zone model while keeping a moderate computational cost.
\end{abstract}

Keywords cohesive zone model $\cdot$ finite elements $\cdot$ time-integration scheme

\section{Introduction}

Cohesive zone models have been introduced in the late $50 \mathrm{~s}[1,2,11]$. They can be applied to a large range of materials (concrete, steel, etc...) and fracture processes (brittle fracture, ductile fracture, fatigue, dynamic fracture) and they can be easily enriched with more complex physical behaviors (contact and friction after decohesion, corrosion, etc...). Moreover, cohesive zone models fit quite well within the

This work was partly supported by Electricité de France (EdF R\&D) through a CIFRE PhD fellowship.

D. Doyen

Université Paris-Est, Laboratoire d'Analyse et de Mathématiques Appliquées (UMR 8050), UPEMLV, UPEC, CNRS, F-77454, Marne-la-Vallée, France

E-mail: david.doyen@univ-mlv.fr

A. Ern

Université Paris-Est, CERMICS, Ecole des Ponts ParisTech, 77455 Marne la Vallée Cedex 2, France

E-mail: ern@cermics.enpc.fr

S. Piperno

Université Paris-Est, CERMICS, Ecole des Ponts ParisTech, 77455 Marne la Vallée Cedex 2,

France

E-mail: serge.piperno@enpc.fr 
framework of finite elements. For all these reasons, they are now widely used in engineering simulations. A cohesive zone model can describe the mechanical forces along a fracture - it can be simply viewed as a boundary condition. The interface forces depend at least on the opening (displacement jump at the interface). In a typical cohesive zone model, the separation occurs at the interface only after a critical stress has been reached. When the separation has occurred, cohesive forces remain. These forces decrease when the opening increases and tend to vanish (softening behavior). Physically, the cohesive forces represent the weakening of the material in the fracture process zone ahead of the crack tip. Furthermore, real cracks cannot experience self-healing in general. To take into account this irreversibility, one can introduce a history parameter, such as the maximal opening. For quasi-static fracture, cohesive zone models depending on opening and maximal opening are well established and in good agreement with experiments. In the dynamic case, numerical simulations with such cohesive zone models predict often crack speeds far higher than those observed in the experiments. For instance, for mode-I fracture in brittle materials, numerical crack tip speeds are close to the Rayleigh wave speed, while experimental crack tip speeds nearly reach half of this value (see for instance $[22,20]$ ). To remedy this, cohesive zone models depending on the opening rate have been designed [21,27]. Such models are called rate-dependent.

Since the crack tip speed is high, typically of the same order as the wave speeds, small time steps are needed to capture accurately the fracture phenomenon. Therefore, it seems natural to consider an explicit time-integration scheme. For cohesive zone models in which the interface forces are related to the opening by a classical function, the use of an explicit time-integration scheme is straightforward [26]. However, in most cohesive zone models, the interface forces are not related to the opening by a classical function, but by a set-valued map. Indeed, most cohesive zone models feature perfect initial adhesion, contact or rigid unloading. There are two main difficulties in using fully explicit schemes in such a context. Firstly, the interface forces are not defined for negative normal opening (see Figure 1 , left). Secondly, the interface tangential forces are discontinuous with respect to the tangential opening (see Figure 1, right), and this can cause oscillations. A first option consists in regularizing the set-valued map to turn it into a singlevalued map. Unfortunately, the regularization of a non-interpenetration condition deteriorates substantially the stability condition of explicit schemes (the penalty contact condition introduces in the model an artificial stiffness larger than the material stiffness). Moreover, replacing a discontinuity by a very stiff slope does not really solve the problem. Alternatively, ad hoc procedures have also been developed to treat a few specific cases of set-valued interface forces: allowing the separation only after a failure criterion has been reached $[5,19,12]$, a posteriori enforcement of the non-interpenetration condition [5,12], tolerance parameter on the tangential opening [12]. For complex cohesive zone models with several set-valued parts, the combined use of these procedures generally becomes quite intricate, or even unfeasible.

In the present work, we focus on dynamic fracture models where the material can only crack along a prescribed surface (fracture interface). In other words, the crack path is known in advance. This assumption may appear as a limitation. However, fracture models predicting the crack path are still quite challenging for industrial applications. Moreover, for a large range of applications (interfacial crack, 
small propagation crack), postulating a priori the crack path is reasonable (see [16] for further discussion). We assume that the bulk behavior is governed by the linear elastodynamic equations and that the separation process at the fracture interface obeys a cohesive zone model. We consider a generic cohesive zone model depending on the opening, the opening rate, and the maximal opening. This generic model encompasses most of the usual cohesive zone models. Space semi-discretization is achieved using $P_{1}$ finite elements. We propose time-integration schemes that combine a central difference scheme with a partially or fully implicit treatment of the interface forces. The central difference scheme is a standard scheme for elastodynamics [15]. The implicit treatment of the interface forces provides a general and robust way of treating the set-valued cohesive zone model. In order to keep a moderate computational cost, we use lumping techniques for the mass term and the interface forces. We thus obtain quasi-explicit methods: at each time step, the displacements of the nodes in the interior of the domain are computed in an explicit way, while the displacements of each node at the interface are computed by solving a small nonlinear problem (this can generally be achieved in an analytical way). First, we consider a fully implicit treatment of the interface forces. However, staggering in time the force at the fracture interface can have a sizeable effect on the energy behavior and the accuracy of the time-integration scheme. Consequently, we propose a second time-integration scheme, in which the interface forces are split into a set-valued monotone part and a single-valued softening part. The former is treated in an implicit way, the latter in an explicit way. This improves the accuracy and the energy behavior. Note that some of the ad hoc procedures described above can be loosely interpreted as an implicit treatment of the set valued part of the cohesive zone model (failure criterion, a posteriori enforcement of the contact condition).

We begin by presenting the generic cohesive zone model and examples which fit into this framework (Section 2). We then formulate the continuous problem of dynamic fracture (Section 3). Sections 4 and 5 are devoted to the finite element discretization in space and to the time-integration schemes, respectively. We discuss the implementation of the schemes in Section 6. Finally, numerical results are presented in Section 7, and conclusions are drawn in Section 8.

\section{Cohesive zone model}

We consider a generic cohesive zone model. The forces at the fracture interface are described by a set-valued map which depends on the opening, the opening rate, and the maximal effective opening (the notion of effective opening is defined below).

\subsection{Generic model}

Let $(\cdot, \cdot)$ denote the usual scalar product in $\mathbb{R}^{n}(n \geq 1)$ and let $|\cdot|$ denote the corresponding Euclidean norm. Let $\mathcal{P}\left(\mathbb{R}^{n}\right)$ denote the set of all subsets of $\mathbb{R}^{n}$. In a $d$-dimensional problem $(d=2$ or $d=3)$, the cohesive zone model is characterized by a set-valued map $R: \mathbb{R}^{d} \times \mathbb{R}^{d} \times \mathbb{R}^{d} \rightarrow \mathcal{P}\left(\mathbb{R}^{d}\right)$. The arguments of $R$ are the maximal effective opening, the opening rate, and the opening, respectively. For each 
triplet $(\delta, z, p)$, the map $R(\delta, z, p)$ yields a set of vectors, which are the admissible interaction forces. The first component of $\lambda \in R(\delta, z, p)$ is the normal force at the interface and the second and third ones are the tangential forces. For an opening $p \in \mathbb{R}^{3}$ (resp. $p \in \mathbb{R}^{2}$ ), the effective opening is defined as $\bar{\delta}(p)=\left(p_{1},\left|p_{2}\right|,\left|p_{3}\right|\right)$ (resp. $\left.\bar{\delta}(p)=\left(p_{1},\left|p_{2}\right|\right)\right)$.

Since a cohesive zone model describes a softening behavior, the set-valued map $R$ is not monotone with respect to the opening. However, the slope of the softening part of $R$ is assumed to be bounded. This assumption is, in particular, useful to establish the well-posedness of our first time-integration scheme (see Proposition 1).

Assumption 1 The operator $R$ satisfies the following one-sided Lipschitz condition: there is a real number $C_{L}$ such that, for all $\delta \in \mathbb{R}^{d}$, for all $z \in \mathbb{R}^{d}$, for all $p, q \in \mathbb{R}^{d}$,

$$
\left(\lambda_{p}-\lambda_{q}, p-q\right) \geq-C_{L}|p-q|^{2}, \quad \forall \lambda_{p} \in R(\delta, z, p), \forall \lambda_{q} \in R(\delta, z, q) .
$$

The present generic model encompasses for instance the Camacho-Ortiz law [5] and the Talon-Curnier law [23], but not the rectangular law (because of the infinite slope of the softening part).

Remark 1 In most cohesive zone models, the operator $R(\delta, z, \cdot)$ is built as the differential (in a generalized sense) of an energy. This operator being non-monotone, the associated cohesive energy is non-convex.

Remark 2 After decohesion, contact closure can lead to friction phenomena at the interface, which can play an important role in the fracture process. Cohesive zone models including friction have been proposed for instance in [18]. The friction force is generally a monotone set-valued function of the tangential velocity at the interface and could be easily added to our generic model.

\subsection{Examples}

This section collects some examples of cohesive zone models fitting the above framework. The first two examples can be viewed as simplified variants of the Camacho-Ortiz law [5].

A reversible triangular model with uncoupled normal and tangential forces This model depends on the opening and prescribes uncoupled normal and tangential interface forces. It relies on two parameters: $\sigma_{c}$, the maximal cohesive force, and $d_{c}$, the critical opening. It can be represented by a set-valued map $R: \mathbb{R}^{d} \rightarrow \mathcal{P}\left(\mathbb{R}^{d}\right)$ whose components are independent. The normal component $R_{1}: \mathbb{R} \rightarrow \mathcal{P}(\mathbb{R})$ is such that

$$
R_{1}(p):= \begin{cases}\left(-\infty, \sigma_{c}\right] & \text { if } p=0 \\ \sigma_{c}\left(1-\frac{p}{d_{c}}\right) & \text { if } 0<p \leq d_{c} \\ 0 & \text { if } d_{c}<p \\ \emptyset & \text { if } p<0\end{cases}
$$




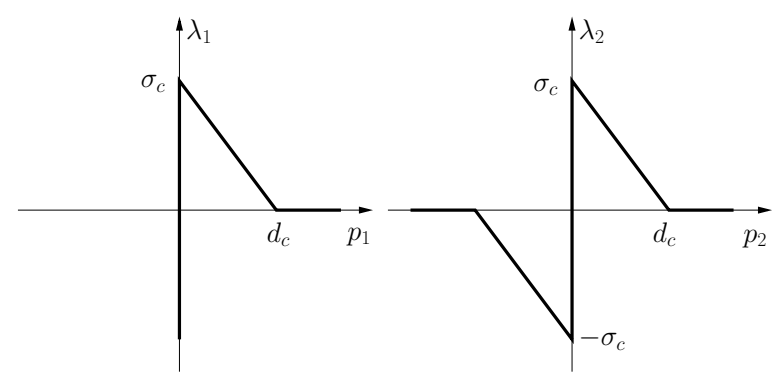

Fig. 1 Triangular model with uncoupled normal and tangential forces. Normal force (left). Tangential force (right).

For simplicity, in the definition of cohesive zone models, a singleton $\{x\}$ is simply denoted $x$. The tangential components $R_{2}: \mathbb{R} \rightarrow \mathcal{P}(\mathbb{R})$ and $R_{3}: \mathbb{R} \rightarrow \mathcal{P}(\mathbb{R})$ are such that

$$
R_{2}(p)=R_{3}(p):= \begin{cases}0 & \text { if } p<-d_{c}, \\ -\sigma_{c}\left(1+\frac{p}{d_{c}}\right) & \text { if }-d_{c} \leq p<0, \\ {\left[-\sigma_{c}, \sigma_{c}\right]} & \text { if } p=0, \\ \sigma_{c}\left(1-\frac{p}{d_{c}}\right) & \text { if } 0<p \leq d_{c}, \\ 0 & \text { if } d_{c}<p .\end{cases}
$$

This model is represented in Figure 1. It is easy to check that this model satisfies Assumption 1 with $C_{L}=\sigma_{c} / d_{c}$. Moreover, energies $\Psi_{1}: \mathbb{R}^{+} \rightarrow \mathbb{R}, \Psi_{2}: \mathbb{R} \rightarrow \mathbb{R}$, and $\Psi_{3}: \mathbb{R} \rightarrow \mathbb{R}$ can be associated with this model, namely

$$
\Psi_{1}(p):= \begin{cases}\sigma_{c} p\left(1-\frac{p}{2 d_{c}}\right) & \text { if } 0 \leq p \leq d_{c} \\ \frac{1}{2} \sigma_{c} d_{c} & \text { if } d_{c}<p\end{cases}
$$

and

$$
\Psi_{2}(p)=\Psi_{3}(p):= \begin{cases}\frac{1}{2} \sigma_{c} d_{c} & \text { if } p<-d_{c}, \\ -\sigma_{c} p\left(1+\frac{p}{2 d_{c}}\right) & \text { if }-d_{c} \leq p<0, \\ \sigma_{c} p\left(1-\frac{p}{2 d_{c}}\right) & \text { if } 0 \leq p \leq d_{c}, \\ \frac{1}{2} \sigma_{c} d_{c} & \text { if } d_{c}<p .\end{cases}
$$

An irreversible triangular model with only normal force This model depends on the normal opening $p$ and maximal effective normal opening $\delta$ and prescribes only the normal force. Moreover, it is irreversible with a linear unloading. As the previous model, it involves two parameters: $\sigma_{c}$, the maximal cohesive force, and $d_{c}$, the critical opening. It can be represented by the set-valued map $R_{1}^{i r r}: \mathbb{R} \times \mathbb{R} \rightarrow \mathcal{P}(\mathbb{R})$ 


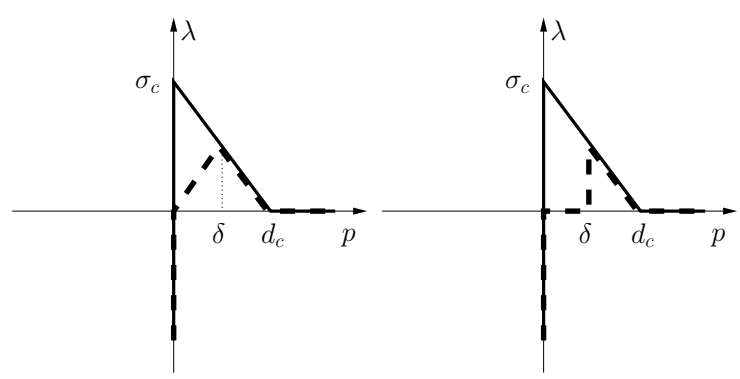

Fig. 2 An irreversible triangular model with only normal force. Linear unloading (left). Rigid unloading (right).

such that

$$
R_{1}^{i r r}(\delta, p):= \begin{cases}\left(-\infty, \sigma_{c}\right] & \text { if } \delta=p=0, \\ (-\infty, 0] & \text { if } 0=p<\delta, \\ \sigma_{c}\left(1-\frac{\delta}{d_{c}}\right) \frac{p}{\delta} & \text { if } 0<p \leq \delta \leq d_{c}, \\ \sigma_{c}\left(1-\frac{p}{d_{c}}\right) & \text { if } 0<\delta<p \leq d_{c}, \\ 0 & \text { if } d_{c}<p, 0 \leq \delta, \\ 0 & \text { if } d_{c}<\delta, 0 \leq p, \\ \emptyset & \text { otherwise. }\end{cases}
$$

This model is represented in Figure 2, left. It satisfies Assumption 1 with $C_{L}=$ $\sigma_{c} / d_{c}$. A common variant of this model consists in replacing the linear unloading with a rigid unloading (Figure 2 , right). An energy $\Psi_{1}^{i r r}: \mathbb{R}^{+} \times \mathbb{R}^{+} \rightarrow \mathbb{R}$ and a dissipated energy $\tilde{\Psi}_{1}^{\text {irr }}: \mathbb{R}^{+} \rightarrow \mathbb{R}$ can be associated with the irreversible model with linear unloading. They are defined as follows:

$$
\begin{gathered}
\Psi_{1}^{i r r}(\delta, p):= \begin{cases}\sigma_{c}\left(-\frac{\delta}{2}+p-\frac{p^{2}}{2 d_{c}}\right) & \text { if } 0<\delta<p \leq d_{c}, \\
\sigma_{c}\left(1-\frac{\delta}{d_{c}}\right) \frac{p^{2}}{2 \delta} & \text { if } 0<p \leq \delta \leq d_{c}, \\
\frac{1}{2} \sigma_{c} d_{c} & \text { if } d_{c}<p, \delta=0, \\
0 & \text { if } d_{c}<\delta, 0 \leq p,\end{cases} \\
\tilde{\Psi}_{1}^{i r r}(\delta):= \begin{cases}\frac{1}{2} \sigma_{c} \delta & \text { if } 0 \leq \delta \leq d_{c}, \\
\frac{1}{2} \sigma_{c} d_{c} & \text { if } d_{c}<\delta .\end{cases}
\end{gathered}
$$

A rate-dependent triangular model with only normal force [27] This model depends on the normal opening $p$ and the normal opening rate $z$, and prescribes only the normal interface force. It relies on three parameters: $\sigma_{c}$, the maximal cohesive force, $d_{c}$, the critical opening, and $\eta$, a viscosity parameter. It can be represented by the set-valued map $R_{1}^{\text {visc }}: \mathbb{R} \times \mathbb{R} \rightarrow \mathcal{P}(\mathbb{R})$ such that

$$
R_{1}^{v i s c}(z, p):= \begin{cases}\left(-\infty, \sigma_{c}\right] & \text { if } p=0 \\ \sigma_{c}\left(1-\frac{p}{d_{c}\left(1+\eta z^{+}\right)}\right) & \text {if } 0<p<d_{c}\left(1+\eta z^{+}\right), \\ 0 & \text { if } d_{c}\left(1+\eta z^{+}\right) \leq p, \\ \emptyset & \text { otherwise }\end{cases}
$$




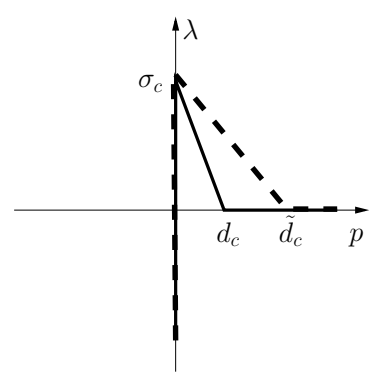

Fig. 3 A rate-dependent triangular model with only normal force $\left(\tilde{d}_{c}:=d_{c}\left(1+\eta z^{+}\right)\right)$.

where $z^{+}$denotes the positive part of $z$. This model is represented in Figure 3. It satisfies Assumption 1 with $C_{L}=\sigma_{c} / d_{c}$.

\subsection{Link with Griffith's model}

It is possible to make a link between cohesive zone models and Griffith's models. When cohesive forces act over a sufficiently short range, the stress fields near the crack tip are equivalent in both models. Furthermore, the material parameter used in Griffith's model, the fracture toughness $G_{c}$, is equal to the energy needed to completely open the crack in the cohesive zone model. A formal argument for this asymptotic analysis can be found in [25] and a rigorous proof for a simple model in [17]. The fracture toughness corresponding to the rate-independent triangular models (presented above) is

$$
G_{c}=\frac{1}{2} \sigma_{c} d_{c}
$$

In the rate-dependent triangular model, the fracture toughness increases with the opening rate.

\section{Continuous problem}

We now formulate the governing equations of the dynamic fracture problem.

\subsection{Geometry}

We consider a domain $\Omega \subset \mathbb{R}^{d}(d=2$ or $d=3)$ and we assume that the crack can only appear on a $(d-1)$-dimensional smooth surface $\Gamma$ (see Figure 4$)$. We call $\Gamma$ the fracture interface. We set $\tilde{\Omega}:=\Omega \backslash \bar{\Gamma}$. We can fix an orientation and define two sides for $\Gamma$, a positive side and a negative side. Let $v: \Omega \rightarrow \mathbb{R}^{d}$ be a displacement field. The trace of $v$ on the positive side is denoted $v^{+}$, the trace on the negative side is denoted $v^{-}$. We denote $\nu$ the unit normal vector to $\Gamma$ pointing to the positive side. We define two tangential unit vectors $\tau_{1}$ and $\tau_{2}$, so that $\left(\nu, \tau_{1}, \tau_{2}\right)$ forms a local direct orthonormal basis (obviously, for $d=2$, only 


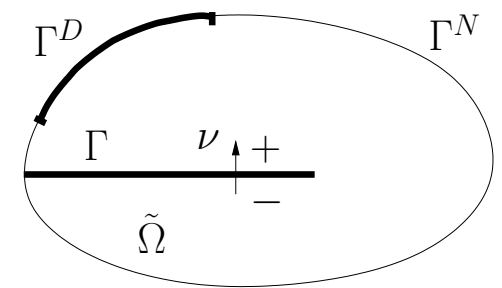

Fig. 4 Geometric setup.

one tangential vector is considered). The displacement jump of $v$ at the interface is defined as

$$
\llbracket v \rrbracket=v^{+}-v^{-} .
$$

To define the interface forces, we take into account the local orientation of the interface by introducing the rotation matrix $Q$ transforming the canonical basis of $\mathbb{R}^{d}$ into $\left(\nu, \tau_{1}, \tau_{2}\right)$.

\subsection{Governing equations}

The material is supposed to be linear isotropic elastic with Young modulus $E$, Poisson ratio $\nu_{P}$, and mass density $\rho$. The elasticity tensor is denoted $\mathcal{A}$. An external load $f$ is applied to the body. Let $u: \Omega \times(0, T) \rightarrow \mathbb{R}^{d}, \epsilon(u): \Omega \times(0, T) \rightarrow$ $\mathbb{R}^{d, d}$, and $\sigma(u): \Omega \times(0, T) \rightarrow \mathbb{R}^{d, d}$ be the displacement field, the linearized strain tensor, and the stress tensor, respectively. Denoting time-derivatives by dots, the momentum conservation equation reads

$$
\rho \ddot{u}-\operatorname{div} \sigma=f, \quad \sigma=\mathcal{A}: \epsilon, \quad \epsilon=\frac{1}{2}\left(\nabla u+{ }^{\mathrm{T}} \nabla u\right) \quad \text { in } \tilde{\Omega} \times(0, T) .
$$

The boundary $\partial \Omega$ of $\Omega$ is partitioned into two disjoint subsets $\Gamma^{D}$ and $\Gamma^{N}$. Dirichlet and Neumann conditions are prescribed on $\Gamma^{D}$ and $\Gamma^{N}$, respectively,

$$
u=u_{D} \quad \text { on } \Gamma^{D} \times(0, T), \quad \sigma \cdot \nu=f_{N} \quad \text { on } \Gamma^{N} \times(0, T) .
$$

On $\Gamma$, the cohesive law is enforced

$$
\sigma\left(u^{-}\right) \cdot \nu=-\sigma\left(u^{+}\right) \cdot \nu=: \lambda, \quad \lambda \in{ }^{\mathrm{T}} Q R(\delta, Q \llbracket \dot{u} \rrbracket, Q \llbracket u \rrbracket) .
$$

The maximal effective opening $\delta$ is defined, for $t>0$, by

$$
\delta(t)=\sup _{s \in[0, t)} \bar{\delta}(Q \llbracket u(s) \rrbracket) .
$$

At the initial time, the displacement, the velocity, and the maximal effective opening are prescribed:

$$
u(0)=u^{0}, \quad \dot{u}(0)=v^{0}, \quad \delta(0)=\delta^{0} .
$$

Equations (3)-(5) can be written in a variational form: seek $u$ such that, for all test function $v$,

$$
\int_{\tilde{\Omega}} \rho \ddot{u} \cdot v+\int_{\tilde{\Omega}} \epsilon(u): \mathcal{A}: \epsilon(v)=\int_{\tilde{\Omega}} f(t) \cdot v+\int_{\Gamma^{N}} f_{N}(t) \cdot v-\int_{\Gamma} \lambda \cdot \llbracket v \rrbracket,
$$

where $\lambda \in{ }^{\mathrm{T}} Q R(\delta, Q \llbracket \dot{u} \rrbracket, Q \llbracket u \rrbracket)$. 
3.3 Mathematical aspects

The mathematical analysis of Problem (3)-(7) is beyond the scope of the present work. However, let us mention some related results.

- In the quasi-static case with a reversible cohesive zone model (with perfect adhesion or not), the existence is proven. The solution is in general not unique [10].

- In the quasi-static case with an irreversible cohesive zone model, the existence of a solution is proven in $[8,4]$.

- In the dynamic case, it should be possible to prove, using compactness arguments similarly to [9], existence for a visco-elastic material and a reversible cohesive law with perfect adhesion, and even to prove existence and uniqueness for an elastic material and a regularized cohesive law.

\subsection{Length and time scales}

In order to capture accurately a phenomenon with numerical simulations, it is important to choose a time step and a mesh size which resolve its time and length scales. In a dynamic fracture problem, the relevant length scale is the length $l_{c o h}$ of the cohesive zone (the part of the interface which is not completely cracked and where cohesive forces act). The relevant time scale is the crack tip speed divided by the cohesive zone length. Several methodologies have been proposed in the literature to estimate the cohesive zone length; see [24] and references therein. They all yield, in the case of plane strain and triangular cohesive zone models, an estimation of the form

$$
l_{c o h}=M \frac{E}{1-\nu_{P}^{2}} \frac{G_{c}}{\sigma_{c}^{2}},
$$

where $M$ is a parameter close to 1 . For an isotropic linear elastic material and a Griffith model of fracture, a theoretical analysis predicts that the limiting crack tip speed for a mode-I fracture is the Rayleigh wave speed [13,3]. For mode-II and mode-III fractures, the limiting speeds are the dilatational wave speed and the shear wave speed, respectively $[13,3]$. The dilatational and shear wave speeds are given by the following formulae:

$$
c_{d}=\sqrt{\frac{E\left(1-\nu_{P}\right)}{\rho\left(1+\nu_{P}\right)\left(1-2 \nu_{P}\right)}}, \quad c_{s}=\sqrt{\frac{E}{2 \rho\left(1+\nu_{P}\right)}} .
$$

The Rayleigh wave speed can be estimated by the following expression [13]

$$
c_{R} \approx c_{s} \frac{0.862+1.14 \nu_{P}}{1+\nu_{P}}
$$

Rate-dependent cohesive zone models involve an additional time scale, linked to the opening rate and generally smaller than the time scale linked to the crack tip speed. At least in the quasi-static evolution, analytical estimations of the opening time are provided in [7]. 


\section{Finite element discretization}

In this section, we describe the space approximation of the dynamic fracture problem. Linear finite elements are used together with lumping of the mass term and the interface forces.

\subsection{Finite element spaces}

In $2 \mathrm{D}$ (resp. in $3 \mathrm{D}$ ), the domain $\Omega$ is approximated by a polygon (resp. a polyhedron) $\Omega_{h}$ and the interface $\Gamma$ by a polygonal curve (resp. a polygon) $\Gamma_{h}$. The domain $\Omega_{h}$ is meshed with triangles (resp. tetrahedra) conforming to the interface $\Gamma_{h}$. Let $\mathcal{T}_{h}$ denote the mesh over $\Omega_{h}$ and let $\mathcal{F}_{h}$ collect the faces located on $\Gamma_{h}$. Let $\tilde{\Omega}_{h}=\Omega_{h} \backslash \overline{\Gamma_{h}}$. Let $\left\{x_{i}\right\}_{i \in \mathcal{N}}$ be the nodes of the mesh $\mathcal{T}_{h}$ where $\mathcal{N}$ collects the node indices. Let $\mathcal{N}^{D}$ be the indices of nodes where a Dirichlet condition is enforced and by $\mathcal{N}^{c}$ the indices of nodes lying on $\Gamma_{h}$. The displacements are approximated with $P_{1}$ finite elements:

$$
\mathcal{V}_{h}=\left\{v_{h} \in C^{0}\left(\tilde{\Omega}_{h}\right)^{d} ; v_{h \mid T} \in\left(\mathbb{P}_{1}\right)^{d}, \forall T \in \mathcal{T}_{h}, \text { and } v_{h}\left(x_{i}\right)=0, \forall i \in \mathcal{N}^{D}\right\} .
$$

Note that functions in $\mathcal{V}_{h}$ can be discontinuous across $\Gamma_{h}$. We consider the Lagrange nodes of $\mathcal{V}_{h}$ and denote them $\left\{\xi_{i}\right\}_{i \in \mathcal{N}^{L}}$ where $\mathcal{N}^{L}$ collects the corresponding node indices. The Lagrange nodes are not exactly the mesh nodes $\left\{x_{i}\right\}_{i \in \mathcal{N}}$ because of the discontinuity at the interface. Specifically, for each mesh node $x_{i}$ lying on $\Gamma_{h}$, there are two Lagrange nodes $\xi_{i^{+}}$and $\xi_{i^{-}}$. For all $i \in \mathcal{N}^{c}$, for all $v_{h} \in \mathcal{V}_{h}$, we set

$$
v_{h}\left(\xi_{i^{+}}\right):=v_{h}^{+}\left(x_{i}\right), \quad v_{h}\left(\xi_{i^{-}}\right):=v_{h}^{-}\left(x_{i}\right) .
$$

The cohesive forces are also approximated by $P_{1}$ finite elements,

$$
\mathcal{L}_{h}=\left\{\lambda_{h} \in C^{0}\left(\overline{\Gamma_{h}}\right)^{d} ; \lambda_{h \mid F} \in\left(\mathbb{P}_{1}\right)^{d}, \forall F \in \mathcal{F}_{h}\right\} .
$$

At each node $x_{i}$ lying on $\overline{\Gamma_{h}}$, we define normal and tangential unit vectors $\left(\nu_{i}, \tau_{1 i}, \tau_{2 i}\right)$ forming a direct orthonormal basis. Let $Q_{i}$ be the associated rotation matrix. We define also the set-valued operator $R_{i}$ :

$$
R_{i}(\cdot, \cdot, \cdot)={ }^{\mathrm{T}} Q_{i} R\left(\cdot, Q_{i} \cdot, Q_{i} \cdot\right) .
$$

\subsection{Lumping of the mass term and the cohesive term}

The mass term and the cohesive forces term are lumped. Mass lumping is usual with explicit time-integration schemes. It yields an easy-to-invert mass matrix at each time step, while improving the CFL condition [15]. A way of lumping the mass term is to evaluate it with an approximate quadrature whose Gauss points are the nodes of the finite element space. For $P_{1}$ finite elements, it is usual to use the following quadrature formula (in dimension $d$ )

$$
\int_{T} f \approx \sum_{i=1}^{d+1} \frac{|T|}{d+1} f\left(\alpha_{i}\right),
$$


where $|T|$ is the measure of the simplex $T$ and $\left\{\alpha_{i}\right\}_{1 \leq i \leq d+1}$ its vertices. This quadrature is second-order accurate. The lumped mass term $\hat{m}_{h}: \mathcal{V}_{h} \times \mathcal{V}_{h} \rightarrow \mathbb{R}$ is built with this quadrature by setting

$$
\hat{m}_{h}\left(v_{h}, w_{h}\right)=\sum_{i \in \mathcal{N}^{L}} \mu_{i} v_{h}\left(\xi_{i}\right) \cdot w_{h}\left(\xi_{i}\right)
$$

where

$$
\mu_{i}=\sum_{T \in \mathcal{T}_{i}} \rho|T| /(d+1)
$$

$\mathcal{T}_{i}$ being the set of elements for which $\xi_{i}$ is a vertex. The lumped cohesive term $\hat{b}_{h}: \mathcal{L}_{h} \times \mathcal{V}_{h} \rightarrow \mathbb{R}$ is such that

$$
\hat{b}_{h}\left(\lambda_{h}, w_{h}\right)=\sum_{i \in \mathcal{N}^{c}} \beta_{i} \lambda_{h}\left(x_{i}\right) \cdot\left(v_{h}^{-}\left(x_{i}\right)-v_{h}^{+}\left(x_{i}\right)\right),
$$

where

$$
\beta_{i}=\sum_{F \in \mathcal{F}_{i}}|F| / d
$$

$\mathcal{F}_{i}$ being the set of faces for which $x_{i}$ is a vertex and $|F|$ the measure of the face $F$. Finally, the stiffness term $a_{h}: \mathcal{V}_{h} \times \mathcal{V}_{h} \rightarrow \mathbb{R}$ and the external force term $l_{h}:[0, T] \times \mathcal{V}_{h} \rightarrow \mathbb{R}$ are built in a standard way, namely

$$
\begin{gathered}
a_{h}\left(v_{h}, w_{h}\right):=\int_{\tilde{\Omega}_{h}} \epsilon\left(v_{h}\right): \mathcal{A}: \epsilon\left(w_{h}\right), \\
l_{h}\left(t, v_{h}\right):=\int_{\tilde{\Omega}_{h}} f(t) \cdot v_{h}+\int_{\Gamma^{N}} f_{N}(t) \cdot v_{h},
\end{gathered}
$$

up to quadratures for $l_{h}$. We define the matrices $\hat{M}_{h}, K_{h}$, and $\hat{B}_{h}$ associated with the bilinear forms $\hat{m}_{h}, a_{h}$, and $\hat{b}_{h}$, respectively. We also define $L_{h}(t)$ to be the column vector associated with the linear form $l_{h}(t, \cdot)$. For $u_{h} \in \mathcal{V}_{h}$, we define $U_{h}$ as the column vector whose components are the coordinates of $u_{h}$ in the finite element basis. We denote $N_{V}$ the size of $U_{h}$. We denote $U_{h, i}$ the $d$-dimensional sub-vector associated with the Lagrange node $\xi_{i}$. Similarly, for $\lambda_{h} \in \mathcal{L}_{h}$, we define $\Lambda_{h}$ as the column vector whose components are the coordinates of $\lambda_{h}$ in the finite element basis. We denote $N_{\Lambda}$ the size of $\Lambda_{h}$. We denote $\Lambda_{h, i}$ the $d$-dimensional sub-vector associated with the node $x_{i}$. Finally, we define, for all $i \in \mathcal{N}^{c}$,

$$
\llbracket U_{h} \rrbracket_{i}=U_{h, i^{+}}-U_{h, i^{-}} \quad \text { and } \quad\left\{U_{h}\right\}_{i}=\frac{U_{h, i^{+}}+U_{h, i^{-}}}{2} .
$$

For each $i \in \mathcal{N}^{L}$, we denote respectively $\hat{M}_{h, i}$ and $K_{h, i}$ the $d \times d$ sub-matrices of $\hat{M}_{h}$ and $K_{h}$ associated with the Lagrange node $\xi_{i}$. For each $i \in \mathcal{N}^{c}$, we denote $\hat{B}_{h, i}$ the $d \times d$ sub-matrix of $\hat{B}_{h}$ associated with the node $x_{i}$. We define the setvalued operator $R_{h}: \mathbb{R}^{N_{\Lambda}} \times \mathbb{R}^{N_{\Lambda}} \times \mathbb{R}^{N_{\Lambda}} \rightarrow \mathcal{P}\left(\mathbb{R}^{N_{\Lambda}}\right)$ such that for all $\Delta_{h} \in \mathbb{R}^{N_{\Lambda}}$, $Z_{h} \in \mathbb{R}^{N_{\Lambda}}, P_{h} \in \mathbb{R}^{N_{\Lambda}}$,

$$
\Lambda_{h} \in R_{h}\left(\Delta_{h}, Z_{h}, P_{h}\right) \Longleftrightarrow \Lambda_{h, i} \in R_{i}\left(\Delta_{h, i}, Z_{h, i}, P_{h, i}\right) \forall i \in \mathcal{N}^{c} .
$$


The space semi-discrete problem takes the form

$$
\begin{aligned}
& \hat{M}_{h} \ddot{U}_{h}(t)+K_{h} U_{h}(t)=L_{h}(t)+\hat{B}_{h} \Lambda_{h}(t), \\
& \Lambda_{h}(t) \in R_{h}\left(\Delta_{h}(t), \llbracket \dot{U}_{h}(t) \rrbracket, \llbracket U_{h}(t) \rrbracket\right), \\
& \Delta_{h, i}(t)=\sup _{s \in[0, t)} \bar{\delta}\left(Q_{i} \llbracket U_{h}(s) \rrbracket_{i}\right), \quad \forall i \in \mathcal{N}^{c} .
\end{aligned}
$$

Remark 3 In the present work, we consider $P_{1}$ finite elements. Other types of finite elements can be used, provided an accurate lumping technique is available. For instance, this is the case for $Q_{1}$ elements (see [15]). For $P_{k}$ and $Q_{k}$ elements with $k \geq 2$, the lumping techniques are more subtle (see for instance [6] and references therein).

Remark 4 Because of lumping, even when the nodes of the interface are in the perfect adhesion regime, our discretization is not equivalent to a discretization without interface. Consequently, small spurious wave reflections can occur at the interface in the perfect adhesion regime.

\section{Time-integration schemes}

It remains now to discretize the problem in time. The time-integration schemes we propose are based on the central difference scheme. To begin with, let us recall the main properties of this scheme in the linear elastodynamic case. We then describe and analyze two schemes for the dynamic fracture problem.

\subsection{Central differences for elastodynamics}

For simplicity, the interval $[0, T]$ is divided into equal subintervals of length $\Delta t$. We set $t^{n}=n \Delta t$ and denote $U_{h}^{n}$ the approximation of $U_{h}$ at time $t^{n}$. For the central difference scheme, the discrete velocity and the discrete acceleration are defined respectively as

$$
\dot{U}_{h}^{n}:=\frac{U_{h}^{n+1}-U_{h}^{n-1}}{2 \Delta t} \text { and } \quad \ddot{U}_{h}^{n}:=\frac{U_{h}^{n+1}-2 U_{h}^{n}+U_{h}^{n-1}}{\Delta t^{2}} .
$$

At each time step of the central difference scheme, one seeks $U_{h}^{n+1}$ such that

$$
\frac{1}{\Delta t^{2}} \hat{M}_{h}\left(U_{h}^{n+1}-2 U_{h}^{n}+U_{h}^{n-1}\right)+K_{h} U_{h}^{n}=L_{h}\left(t^{n}\right) .
$$

The central difference scheme exhibits a stability condition (CFL condition) of the form

$$
c_{d} \Delta t \leq O\left(h_{\min }\right),
$$

where $h_{\text {min }}$ is the smallest mesh element diameter. An admissible value of the constant in the CFL condition can be specified in 1D and for structured meshes in higher dimension. The elastic energy, the kinetic energy, and the total energy of the discrete system at time $t^{n}$ are respectively defined by

$$
E_{e l}^{n}:=\frac{1}{2}\left(K_{h} U_{h}^{n}, U_{h}^{n}\right), \quad E_{k i n}^{n}:=\frac{1}{2}\left(\hat{M}_{h} \dot{U}_{h}^{n}, \dot{U}_{h}^{n}\right), \quad E^{n}:=E_{e l}^{n}+E_{k i n}^{n} .
$$


In linear elastodynamics with no forcing term $L_{h}\left(t^{n}\right)$, the central difference scheme does not preserve the energy. Nevertheless, the scheme preserves the following quadratic form, referred to as a shifted energy,

$$
\mathcal{E}^{n}:=E^{n}-\frac{\Delta t^{2}}{8}\left(\hat{M}_{h} \ddot{U}_{h}^{n}, \ddot{U}_{h}^{n}\right)
$$

With a forcing term, the following shifted energy balance holds true:

$$
\mathcal{E}^{n+1}-\mathcal{E}^{n}=\frac{1}{2}\left(L_{h}\left(t^{n+1}\right)+L_{h}\left(t^{n}\right), U_{h}^{n+1}-U_{h}^{n}\right) .
$$

\subsection{Scheme A (fully implicit interface forces)}

The scheme A combines a central difference scheme with an implicit treatment of the interface forces. More precisely, the interface forces are implicit in the opening, while they are explicit in the opening rate and in the maximal effective opening. Scheme A. Seek $U_{h}^{n+1} \in \mathbb{R}^{N_{V}}$ and $\Lambda_{h}^{n+1} \in \mathbb{R}^{N_{\Lambda}}$ such that

$$
\begin{aligned}
& \frac{1}{\Delta t^{2}} \hat{M}_{h}\left(U_{h}^{n+1}-2 U_{h}^{n}+U_{h}^{n-1}\right)+K_{h} U_{h}^{n}=L_{h}\left(t^{n}\right)+\hat{B}_{h} \Lambda_{h}^{n+1}, \\
& \Lambda_{h}^{n+1} \in R_{h}\left(\Delta_{h}^{n}, Z_{h}^{n}, \llbracket U_{h}^{n+1} \rrbracket\right),
\end{aligned}
$$

where, for all $i \in \mathcal{N}^{c}$,

$$
Z_{h, i}^{n}=\frac{\llbracket U_{h}^{n} \rrbracket_{i}-\llbracket U_{h}^{n-1} \rrbracket_{i}}{\Delta t}, \quad \Delta_{h, i}^{n}=\max \left(\Delta_{h, i}^{n-1}, \bar{\delta}\left(Q_{i} \llbracket U_{h}^{n} \rrbracket_{i}\right)\right) .
$$

A way of implementing this scheme will be described in Section 6.1. We now prove that, at each time step, the problem is well-posed under a mild restriction on the time step. We observe that condition (36) below is indeed mild, since for $h_{c}$ and $h_{\min }$ of the same size and small enough, the stability condition (28) of the central difference scheme imposes a more stringent limit on the time step than the well-posedness condition (36).

Proposition 1 (Well-posedness) Problem (32)-(34) has a unique solution under the conditions

$$
\frac{\mu_{i^{+}}}{\Delta t^{2}}>2 C_{L} \beta_{i} \quad \text { and } \quad \frac{\mu_{i^{-}}}{\Delta t^{2}}>2 C_{L} \beta_{i}, \quad \forall i \in \mathcal{N}^{c}
$$

where the coefficients $\mu_{i}$ and $\beta_{i}$ are defined by (16) and (18). For a quasi-uniform mesh, the above condition can be rewritten as

$$
\frac{\Delta t^{2}}{h_{c}}<C
$$

where $h_{c}$ is the mesh size at the interface and $C$ a constant independent of the mesh size. 
Proof The relations (32)-(33) can be recast as an inclusion : seek $U_{h}^{n+1} \in \mathbb{R}^{N_{V}}$ such that

$$
\frac{1}{\Delta t^{2}} \hat{M}_{h} U_{h}^{n+1}-\hat{B}_{h} R_{h}^{n}\left(U_{h}^{n+1}\right) \ni F_{h}^{n}
$$

where $F_{h}^{n}:=\left(1 / \Delta t^{2}\right) \hat{M}_{h}\left(2 U_{h}^{n}-U_{h}^{n-1}\right)-K_{h} U_{h}^{n}+L_{h}\left(t^{n}\right)$ and $R_{h}^{n}\left(U_{h}^{n+1}\right):=$ $R_{h}\left(\Delta_{h}^{n}, Z_{h}^{n}, \llbracket U_{h}^{n+1} \rrbracket\right)$. We are now going to prove that the set-valued operator involved in this inclusion is strongly monotone for $\Delta t$ small enough. Let $U_{h} \in \mathbb{R}^{N_{V}}$ and let $V_{h} \in \mathbb{R}^{N_{V}}$. Let $\Lambda_{h} \in R_{h}^{n}\left(U_{h}\right)$ and $\Theta_{h} \in R_{h}^{n}\left(V_{h}\right)$. Observe that

$$
-\left(\hat{B}_{h} \Lambda_{h}-\hat{B}_{h} \Theta_{h}, U_{h}-V_{h}\right)=\sum_{i \in \mathcal{N}^{c}} \beta_{i}\left(B_{h, i} \Lambda_{h, i}-B_{h, i} \Theta_{h, i}, \llbracket U_{h} \rrbracket_{i}-\llbracket V_{h} \rrbracket_{i}\right) .
$$

Using the one-sided Lipschitz condition (1) and the properties of the rotation matrices $Q_{i}$,

$$
\begin{aligned}
-\left(\hat{B}_{h} \Lambda_{h}-\hat{B}_{h} \Theta_{h}, U_{h}-V_{h}\right) & \geq-\sum_{i \in \mathcal{N}^{c}} \beta_{i} C_{L}\left|\llbracket U_{h} \rrbracket_{i}-\llbracket V_{h} \rrbracket_{i}\right|^{2}, \\
& \geq-\sum_{i \in \mathcal{N}^{c}} 2 \beta_{i} C_{L}\left(\left|U_{h, i^{+}}-V_{h, i^{+}}\right|^{2}+\left|U_{h, i^{-}}-V_{h, i^{-}}\right|^{2}\right) .
\end{aligned}
$$

By definition of the mass matrix,

$$
\left(\hat{M}_{h} U_{h}-\hat{M}_{h} V_{h}, U_{h}-V_{h}\right) \geq \sum_{i \in \mathcal{N}^{L}} \mu_{i}\left|U_{h, i}-V_{h, i}\right|^{2} .
$$

Collecting inequalities (38) and (39) yields

$$
\begin{aligned}
& \frac{1}{\Delta t^{2}}\left(\hat{M}_{h} U_{h}-\hat{M}_{h} V_{h}, U_{h}-V_{h}\right)-\left(\hat{B}_{h} \Lambda_{h}-\hat{B}_{h} \Theta_{h}, U_{h}-V_{h}\right) \geq \\
& \sum_{i \in \mathcal{N}^{L}} \frac{\mu_{i}}{\Delta t^{2}}\left|U_{h, i}-V_{h, i}\right|^{2}-\sum_{i \in \mathcal{N}^{c}} 2 \beta_{i} C_{L}\left(\left|U_{h, i^{+}}-V_{h i^{+}}\right|^{2}+\left|U_{h, i^{-}}-V_{h, i^{-}}\right|^{2}\right) .
\end{aligned}
$$

Therefore, a sufficient condition for the set-valued operator to be strongly monotone is given by (35). For a quasi-uniform mesh, we can rewrite the condition as (36).

It is straightforward to derive the energy balance for Scheme A.

\section{Proposition 2 (Energy balance)}

$\mathcal{E}^{n+1}-\mathcal{E}^{n}=\frac{1}{2}\left(\hat{B}_{h} \Lambda_{h}^{n+2}+\hat{B}_{h} \Lambda_{h}^{n+1}, U_{h}^{n+1}-U_{h}^{n}\right)+\frac{1}{2}\left(L_{h}\left(t^{n+1}\right)+L_{h}\left(t^{n}\right), U_{h}^{n+1}-U_{h}^{n}\right)$.

With this scheme, the work of the interface forces can be positive or negative. As a consequence, we cannot prove the same CFL condition as in the linear elastodynamic case, although we did observe numerically the same CFL condition (see Section 7.1). Perhaps more importantly, it turns out (see again Section 7.1) that decentering in time the interface forces can have an unfavorable impact on the energy behavior and the accuracy of the time-integration scheme. To remedy this, we propose another time-integration scheme in the next section. 


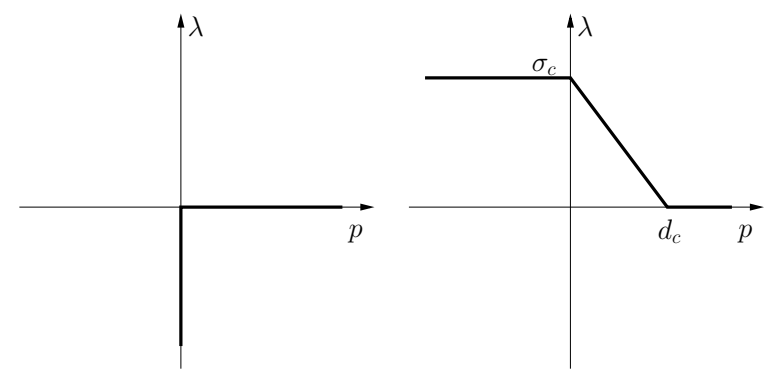

Fig. 5 A decomposition of $R_{1} . P_{1}$ (left) and $\chi_{1}$ (right).

5.3 Scheme B (implicit set-valued part)

The key idea is to split the interface forces into a set-valued monotone part and a single-valued softening part. The former is treated in an implicit way, the latter in an explicit way.

Assumption 2 There exist $P: \mathbb{R}^{d} \times \mathbb{R}^{d} \times \mathbb{R}^{d} \rightarrow \mathcal{P}\left(\mathbb{R}^{d}\right)$ and $\chi: \mathbb{R}^{d} \times \mathbb{R}^{d} \times \mathbb{R}^{d} \rightarrow \mathbb{R}^{d}$ such that

$$
R=P+\chi
$$

Moreover, the operator $P$ is monotone with respect to the opening: for all $\delta \in \mathbb{R}^{d}$, $z \in \mathbb{R}^{d}, p \in \mathbb{R}^{d}, q \in \mathbb{R}^{d}$,

$$
\left(\lambda_{p}-\lambda_{q}, p-q\right) \geq 0, \quad \forall \lambda_{p} \in P(\delta, z, p), \forall \lambda_{q} \in P(\delta, z, q),
$$

and the function $\chi$ is Lipschitz continuous with respect to the opening : there exists $C_{L} \in \mathbb{R}^{+}$such that for all $\delta \in \mathbb{R}^{d}, z \in \mathbb{R}^{d}, p \in \mathbb{R}^{d}, q \in \mathbb{R}^{d}$,

$$
|\chi(\delta, z, p)-\chi(\delta, z, q)| \leq C_{L}|p-q| \text {. }
$$

Of course, the decomposition (42) of $R$ is not unique. In Figures 5, 6, and 7, we present examples of the decomposition (42) for the cohesive zone models presented in Section 2.2. Similarly to (13), the set-valued operator $P_{i}$ and the single-valued function $\chi_{i}$ are defined by

$$
P_{i}(\cdot, \cdot, \cdot)={ }^{\mathrm{T}} Q_{i} P\left(\cdot, Q_{i} \cdot Q_{i} \cdot\right), \quad \chi_{i}(\cdot, \cdot, \cdot)={ }^{\mathrm{T}} Q_{i} \chi\left(\cdot, Q_{i} \cdot Q_{i} \cdot\right) .
$$

Scheme B. Seek $U_{h}^{n+1} \in \mathbb{R}^{N_{V}}$ and $\Lambda_{h}^{n+1} \in \mathbb{R}^{N_{\Lambda}}$ such that

$$
\begin{aligned}
& \frac{1}{\Delta t^{2}} \hat{M}_{h}\left(U_{h}^{n+1}-2 U_{h}^{n}+U_{h}^{n-1}\right)+K_{h} U_{h}^{n}=L_{h}\left(t^{n}\right)+\hat{B}_{h} \Lambda_{h}^{n+1}+\hat{B}_{h} \Theta_{h}^{n}, \\
& \Lambda_{h}^{n+1} \in P_{h}\left(\Delta_{h}^{n}, Z_{h}^{n}, \llbracket U_{h}^{n+1} \rrbracket\right) .
\end{aligned}
$$

where, for all $i \in \mathcal{N}^{c}$,

$$
\Theta_{h, i}^{n}=\chi_{i}\left(\Delta_{h, i}^{n}, Z_{h, i}^{n}, \llbracket U_{h}^{n} \rrbracket_{i}\right),
$$

while $Z_{h}^{n}$ and $\Delta_{h}^{n}$ are defined by (34).

Proposition 3 (Well-posedness) Problem (46)-(48) has a unique solution unconditionally on the time step. 


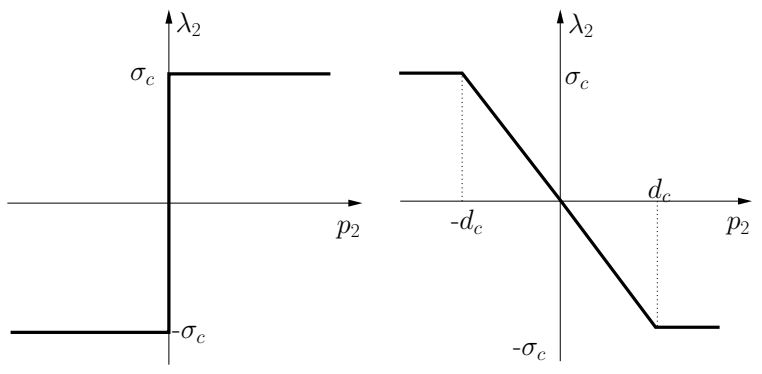

Fig. 6 A decomposition of $R_{2} . P_{2}$ (left) and $\chi_{2}$ (right).
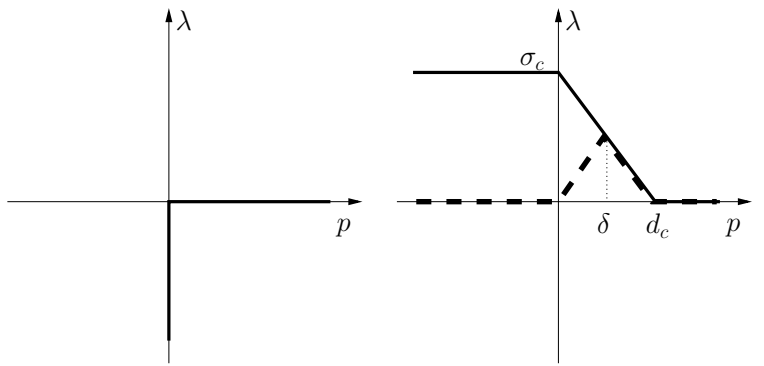

Fig. 7 A decomposition of $R_{1}^{i r r} . P_{1}^{i r r}$ (left) and $\chi_{1}^{\text {irr }}$ (right).

Proof As in the proof of Proposition 3, the problem is recast as a differential inclusion with a strongly monotone operator. Since the operator $P$ is monotone, contrary to the operator $R$, there is no condition on the time step to prove the strong monotonicity.

It is straightforward to derive the energy balance for Scheme B.

\section{Proposition 4 (Energy balance)}

$$
\begin{aligned}
\mathcal{E}^{n+1}-\mathcal{E}^{n}= & \frac{1}{2}\left(\hat{B}_{h} \Lambda_{h}^{n+2}+\hat{B}_{h} \Lambda_{h}^{n+1}, U_{h}^{n+1}-U_{h}^{n}\right) \\
& +\frac{1}{2}\left(\hat{B}_{h} \Theta_{h}^{n+1}+\hat{B}_{h} \Theta_{h}^{n}, U_{h}^{n+1}-U_{h}^{n}\right)+\frac{1}{2}\left(L_{h}\left(t^{n+1}\right)+L_{h}\left(t^{n}\right), U_{h}^{n+1}-U_{h}^{n}\right) .
\end{aligned}
$$

\section{Numerical implementation}

This section briefly describes the main steps to implement Schemes A and B.

\subsection{Scheme A}

Step 1 : Computation of the interior components of $U_{h}^{n+1}$ (explicit step).

Owing to the lumping of the mass matrix, the components of $U_{h}^{n+1}$ corresponding 
to the interior nodes and the components corresponding to the interface nodes can be computed independently. We begin by seeking $U_{h}^{n+1}$ such that

$$
\frac{1}{\Delta t^{2}} \hat{M}_{h}\left(U_{h}^{n+1}-2 U_{h}^{n}+U_{h}^{n-1}\right)+K U_{h}^{n}=L_{h}\left(t^{n}\right) .
$$

This is the standard step in the central difference scheme. Since the mass matrix is lumped, it only requires a matrix-vector multiplication and vector additions. After this step, the interface components of $U_{h}^{n+1}$ are not correct, they will be corrected in the next step. Note that this step uses the standard data structures.

Step 2 : Computation of the interface components of $U_{h}^{n+1}$ (implicit step).

Owing to the lumping of the cohesive forces, the computation of the interface displacements can performed independently at each node. For each $i \in \mathcal{N}^{c}$, we seek $U_{h, i^{+}}^{n+1}$ and $U_{h, i^{-}}^{n+1}$ such that

$$
\begin{aligned}
& \frac{1}{\Delta t^{2}} M_{h, i^{+}} U_{h, i^{+}}^{n+1}+F_{h, i^{+}}^{n}=\hat{B}_{h, i} \Lambda_{h, i}^{n+1}, \\
& \frac{1}{\Delta t^{2}} M_{h, i^{-}} U_{h, i^{-}}^{n+1}+F_{h, i^{-}}^{n}=-\hat{B}_{h, i} \Lambda_{h, i}^{n+1}, \\
& \Lambda_{h, i}^{n+1} \in R_{i}^{n}\left(\llbracket U \rrbracket_{h, i}^{n+1}\right),
\end{aligned}
$$

where $R_{i}^{n}(\cdot)=R_{i}\left(\Delta_{h, i}^{n}, Z_{h, i}^{n}, \cdot\right)$ and $F_{h}^{n}:=\left(1 / \Delta t^{2}\right) \hat{M}_{h}\left(2 U_{h}^{n}-U_{h}^{n-1}\right)-K_{h} U_{h}^{n}+$ $L_{h}\left(t^{n}\right)$. Problem (51)-(53) amounts to seeking $\llbracket U_{h}^{n+1} \rrbracket_{i}$ and $\left\{U_{h}^{n+1}\right\}_{i}$ such that

$$
\begin{aligned}
& \frac{1}{\Delta t^{2}} \llbracket U_{h}^{n+1} \rrbracket_{i}+M_{h, i^{+}}^{-1} F_{h, i^{+}}^{n}-M_{h, i^{-}}^{-1} F_{h, i^{-}}^{n} \in\left(M_{h, i^{+}}^{-1}+M_{h, i^{-}}^{-1}\right) \hat{B}_{h, i} R_{i}^{n}\left(\llbracket U_{h}^{n+1} \rrbracket_{i}\right), \\
& \frac{1}{\Delta t^{2}} M_{h, i^{+}} M_{h, i^{-}}\left\{U_{h}^{n+1}\right\}_{i}+\frac{1}{2}\left(M_{h, i^{+}} F_{h, i^{-}}^{n}+M_{h, i^{-}} F_{h, i^{+}}^{n}\right)=0 .
\end{aligned}
$$

The inclusion can generally be solved in an analytical way. For instance, for the normal component of an irreversible triangular model, we have to solve, at each node, a scalar inclusion of the form: seek $x \in \mathbb{R}$ such that

$$
-\frac{a}{\Delta t^{2}} x+b \in R_{1}(x)
$$

where $a$ and $b$ are given real numbers. Since $R_{1}$ is non-monotone, this problem may have in general several solutions (Figure 8, left). However, for a time step satisfying the condition (35) (thus stiffening the dotted line as in Figure 8, right), the solution is unique.

Step 3 : Update of $\Delta_{h}^{n+1}$ and $Z_{h}^{n+1}$ using the explicit formulae (34).

\subsection{Scheme B}

Step 1 : Computation of the interior components of $U_{h}^{n+1}$ (explicit step). We begin by seeking $U_{h}^{n+1}$ such that

$$
\frac{1}{\Delta t^{2}} \hat{M}_{h}\left(U_{h}^{n+1}-2 U_{h}^{n}+U_{h}^{n-1}\right)+K_{h} U_{h}^{n}=L_{h}\left(t^{n}\right)+\hat{B}_{h} \Theta_{h}^{n} .
$$




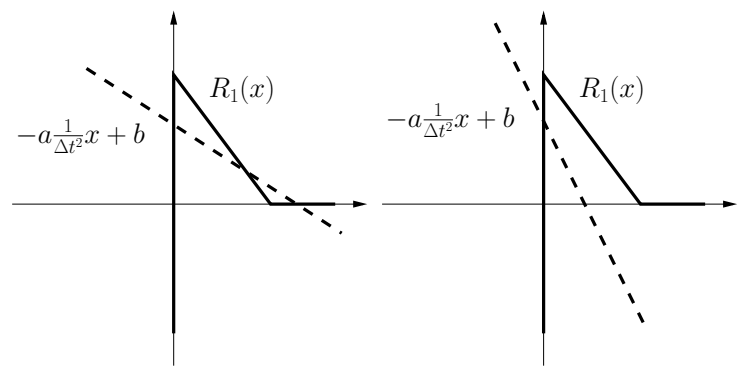

Fig. 8 Computation of the interface components for Scheme A.

Step 2 : Computation of the interface components of $U_{h}^{n+1}$ (implicit step). For each $i \in \mathcal{N}^{c}$, we have to seek $U_{h, i^{+}}^{n+1}$ and $U_{h, i^{-}}^{n+1}$ such that

$$
\begin{aligned}
& \frac{1}{\Delta t^{2}} M_{h, i^{+}} U_{h, i^{+}}^{n+1}+F_{h, i^{+}}^{n}=\hat{B}_{h, i} \Lambda_{h, i}^{n+1}, \\
& \frac{1}{\Delta t^{2}} M_{h, i^{-}} U_{h, i^{-}}^{n+1}+F_{h, i^{-}}^{n}=-\hat{B}_{h, i} \Lambda_{h, i}^{n+1}, \\
& \Lambda_{h, i}^{n+1} \in P_{i}^{n}\left(\llbracket U_{h} \rrbracket_{i}\right),
\end{aligned}
$$

where $P_{i}^{n}(\cdot)=P_{i}\left(\Delta_{h, i}^{n}, Z_{h, i}^{n}, \cdot\right)$ and $F_{h}^{n}:=\left(1 / \Delta t^{2}\right) \hat{M}_{h}\left(2 U_{h}^{n}-U_{h}^{n-1}\right)-K_{h} U_{h}^{n}+$ $L_{h}\left(t^{n}\right)+\hat{B}_{h} \Theta_{h}^{n}$. Problem (58)-(60) amounts to seeking $\llbracket U_{h}^{n+1} \rrbracket_{i}$ and $\left\{U_{h}^{n+1}\right\}_{i}$ such that

$$
\begin{aligned}
& \frac{1}{\Delta t^{2}} \llbracket U_{h}^{n+1} \rrbracket_{i}+M_{h, i^{+}}^{-1} F_{h, i^{+}}^{n}-M_{h, i^{-}}^{-1} F_{h, i^{-}}^{n} \in\left(M_{h, i^{+}}^{-1}+M_{h, i^{-}}^{-1}\right) \hat{B}_{h, i} P_{i}^{n}\left(\llbracket U_{h}^{n+1} \rrbracket_{i}\right), \\
& \frac{1}{\Delta t^{2}} M_{h, i^{+}} M_{h, i^{-}}\left\{U_{h}^{n+1}\right\}_{i}+\frac{1}{2}\left(M_{h, i^{+}} F_{h, i^{-}}^{n}+M_{h, i^{-}} F_{h, i^{+}}^{n}\right)=0 .
\end{aligned}
$$

The inclusion can generally be solved in an analytical way. For instance, for the normal component of an irreversible triangular model (decomposed as in Figure 5), we have to solve, at each node, a scalar inclusion of the form: seek $x \in \mathbb{R}$ such that

$$
-\frac{a}{\Delta t^{2}} x+b \in P_{1}(x)
$$

where $a$ and $b$ are given real numbers. Since $P_{1}$ is monotone, there is a unique solution to this problem (Figure 9).

Step 3 : Update of $\Delta_{h}^{n+1}$ and $Z_{h}^{n+1}$ using the explicit formulae (34).

\section{Numerical simulations}

We perform numerical simulations on $2 \mathrm{D}$ examples with the purpose to illustrate the effectiveness of the quasi-explicit time-integration schemes and to investigate their properties (stability condition on the time step, accuracy). For the first problem, we test the quasi-explicit time-integration schemes A and B, and a fully explicit scheme with a regularized cohesive zone model. For the other problems, 


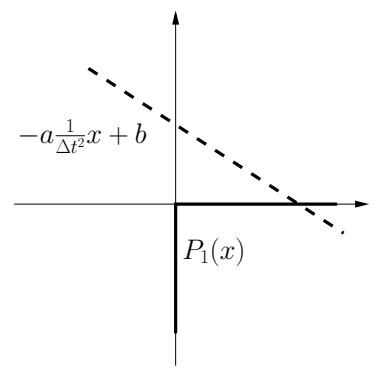

Fig. 9 Computation of the interface components for Scheme B.

we only test Scheme B, which emerges as the most efficient scheme on the first problem. All the simulations have been performed using FREEFEM ++ [14].

\subsection{Mode I fracture}

We consider a mode-I fracture problem (Figure 10). The domain is the rectangle $\Omega=\left(0, L_{x}\right) \times\left(-L_{y}, L_{y}\right)$. The fracture interface is the line $\Gamma=\left(0, L_{x}\right) \times\{0\}$. The cohesive zone model is a reversible triangular model with fracture toughness $G_{c}$ and critical stress $\sigma_{c}$. A constant displacement load is prescribed at the bottom and top edges $\left(u_{y}=u_{D}\right.$ at the top edge and $u_{y}=-u_{D}$ at the bottom edge, with $\left.u_{D}=\epsilon L_{y}\right)$. At the initial time, a precrack of length $L_{p r e}$ is inserted at mid-height on the left edge. Unless otherwise specified, the parameters are $L_{x}=100 \mathrm{~mm}, L_{y}$ $=10 \mathrm{~mm}, L_{\text {pre }}=10 \mathrm{~mm}, \epsilon=0.003, T=14 \mu \mathrm{s}, E=200 \mathrm{GPa}, \nu_{P}=0, \rho=$ $7800 \mathrm{~kg} \cdot \mathrm{m}^{-3}, \sigma_{c}=1.2 \mathrm{GPa}$, and $G_{c}=16000 \mathrm{~N} \cdot \mathrm{m}^{-1}$. The material parameters are representative of those of a steel (except the Poisson ratio which is taken to be zero to facilitate the determination of the initial condition). The wave speeds associated with the above parameters are $c_{d}=5064 \mathrm{~m} \cdot \mathrm{s}^{-1}, c_{s}=3581 \mathrm{~m} \cdot \mathrm{s}^{-1}$, and $c_{R}=3086 \mathrm{~m} \cdot \mathrm{s}^{-1}$. The estimated cohesive zone length and the critical opening are $l_{\text {coh }}=1.963 \mathrm{~mm}$ and $d_{c}=0.0267 \mathrm{~mm}$.

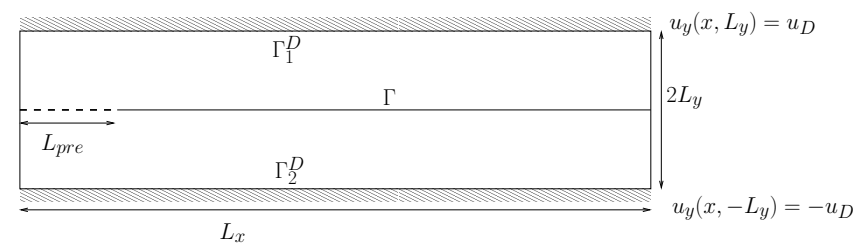

Fig. 10 Mode I fracture. Geometric setup.

Owing to symmetry, numerical simulations are performed only on the upper half part of the domain. This half-domain is uniformly meshed with half-square triangles. We denote $n_{m e s h}$ the number of elements on the large edge of the domain and by $\Delta x$ the length of the elements on this edge. The time step is then 

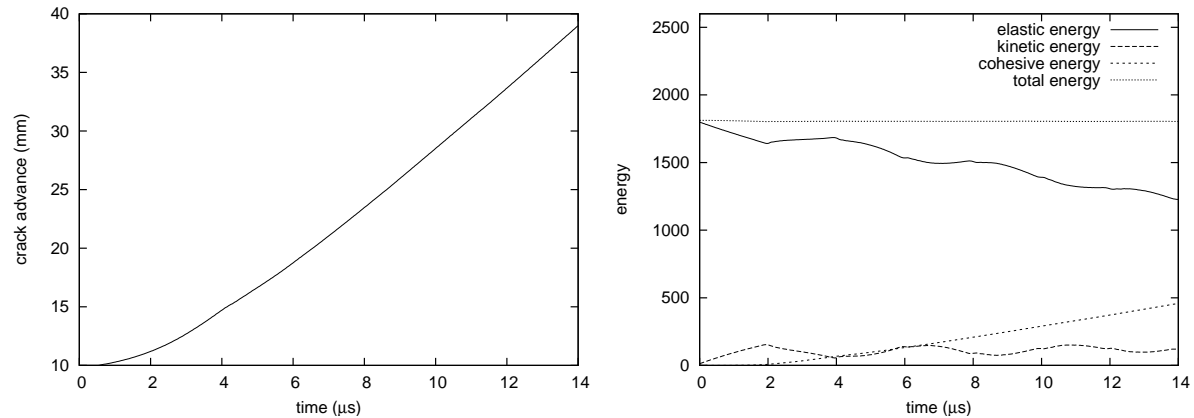

Fig. 11 Mode I fracture. Crack tip advance (left) and energy evolution (right). Scheme B $\left(n_{\text {mesh }}=800, \nu_{C}=1\right)$.

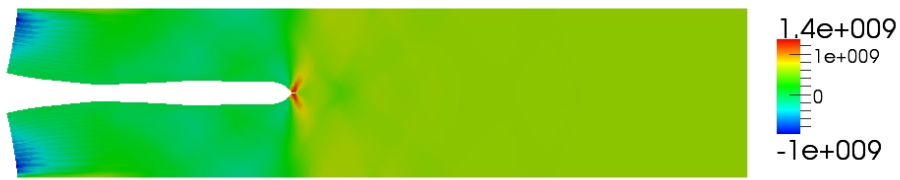

Fig. 12 Mode I fracture. Deformed configuration (enlarged 50 times) and stress $\sigma_{y y}$ at time $T$. Scheme B $\left(n_{\text {mesh }}=800, \nu_{C}=1\right)$.

determined by fixing a value for the Courant number defined as $\nu_{C}:=c_{d} \sqrt{2} \frac{\Delta t}{\Delta x}$. We are interested in the following quantities: crack length, crack tip speed, and energy. We denote $l_{c r}(t)$ the length of the crack at time $t$ and by $v_{c r}\left(t_{1}, t_{2}\right)$ the average crack tip speed between times $t_{1}$ and $t_{2}$. To localize the crack tip, we consider that the interface is cracked when the opening exceeds $0.1 d_{c}$. We denote $E_{e l}(t), E_{k i n}(t), E_{c o h}(t)$, and $E_{t o t}(t)$, the elastic, kinetic, cohesive, and total energy at time $t$, respectively. The cohesive energy is defined as

$$
E_{c o h}(t):=\sum_{i \in \mathcal{N}^{c}} \beta_{i} \Psi_{1}\left(\llbracket U_{h}(t) \rrbracket_{i, 1}\right) .
$$

The total energy is defined as the sum of the elastic, kinetic, and cohesive energy. In the continuous problem, the total energy is expected to be constant (although there is no rigorous proof of this fact). In the different simulations, we observe the following qualitative behavior: the precrack opens, and there is a stress concentration around the precrack tip; as soon as this stress reaches the cohesive critical stress, the crack starts growing; the crack tip speed quickly reaches a limit value (lower than the Rayleigh wave speed). This behavior is illustrated in Figure 11 (crack tip advance and energy evolution) and Figure 12 (deformation and stress fields at time $T$ ).

We investigate the properties of the quasi-explicit time-integration schemes A and $\mathrm{B}$, and a fully explicit scheme with a regularized cohesive zone model (the penalty parameter for the regularization of the non-interpenetration condition is denoted $p_{c}$ ). First of all, we examine the stability condition. We observe numerically that Schemes A and B exhibit the same CFL condition as the central 


\begin{tabular}{|c|c|c|c|}
\hline$p_{c} / C_{L}$ & 10 & 100 & 1000 \\
\hline stability condition & $\nu_{C} \lesssim 0.88$ & $\nu_{C} \lesssim 0.33$ & $\nu_{C} \lesssim 0.11$ \\
\hline
\end{tabular}

Table 1 Mode I fracture. Observed stability condition for a fully explicit scheme with regularized cohesive zone model $\left(n_{\text {mesh }}=200\right)$.

difference scheme on the problem without cohesive zone, namely $\nu_{C} \lesssim 1$. On the contrary, a fully explicit treatment of a regularized cohesive zone model deteriorates the CFL condition (Table 1 ).

Now we compare the accuracy of the different schemes. We choose a mesh size equivalent or finer than the estimated cohesive length. With $\nu_{C}=1$, we observe that Scheme A is not very accurate (Table 2). In particular, the energy balance is quite poor. Since the total energy should remain constant at the value 1800 for the present parameter choice, we infer a relative error of $17.4 \%$ for $n_{m e s h}=200$. By taking a smaller Courant number, the results are improved, leading to a relative error of $5.1 \%$ for $\nu_{C}=0.4$ and $0.9 \%$ for $\nu_{C}=0.1$. In contrast to Scheme A, Scheme B yields accurate results with $\nu_{C}=1$ (Table 3), with, in particular, a relative error on the total energy of $0.7 \%$ for $n_{\text {mesh }}=200$. Moreover, for the crack length and the average speed of the crack tip (for which analytical values are unknown), we observe that, as the grid is refined, the computed values converge faster for Scheme B than for Scheme A, with three significant digits reached by Scheme B for $n_{m e s h}=400$ for the crack length and $n_{m e s h}=200$ for the average speed. Finally, the results of the fully explicit scheme with regularized cohesive zone model are relatively accurate (Table 4), where the lowest relative error on the total energy is $1.3 \%$ for $p_{c} / C_{L}=1000$ and $\nu_{C}=0.11$. However, in addition to a more restrictive stability condition than Scheme B, this scheme does not enforce a perfect adhesion on the sound part of the interface (Figure 13).

\begin{tabular}{|c|c|c|c|c|c|c|c|c|}
\hline$n_{\text {mesh }}$ & $\nu_{C}$ & $l_{\text {coh }} / \Delta x$ & $l_{c r}(T)$ & $v_{c r}(T / 2, T)$ & $E_{e l}(T)$ & $E_{k i n}(T)$ & $E_{c o h}(T)$ & $E_{\text {tot }}(T)$ \\
\hline 100 & 1 & 1.963 & 0.04495 & 2930 & 1388 & 352.3 & 560.1 & 2301 \\
200 & 1 & 3.927 & 0.04376 & 2956 & 1301 & 275.1 & 537.3 & 2113 \\
400 & 1 & 7.854 & 0.04197 & 2845 & 1239 & 194.6 & 510.4 & 1944 \\
800 & 1 & 15.71 & 0.04052 & 2723 & 1226 & 148.7 & 485.7 & 1860 \\
\hline \hline$n_{\text {mesh }}$ & $\nu_{C}$ & $l_{\text {coh }} / \Delta x$ & $l_{c r}(T)$ & $v_{c r}(T / 2, T)$ & $E_{e l}(T)$ & $E_{k i n}(T)$ & $E_{c o h}(T)$ & $E_{\text {tot }}(T)$ \\
\hline 200 & 0.4 & 3.927 & 0.04071 & 2737 & 1240 & 163.8 & 488.9 & 1892 \\
200 & 0.2 & 3.927 & 0.0395 & 2619 & 1233 & 133.2 & 472.3 & 1839 \\
200 & 0.1 & 3.927 & 0.03897 & 2581 & 1232 & 120.3 & 464.1 & 1817 \\
\hline
\end{tabular}

Table 2 Mode I fracture. Numerical results. Scheme A.

Finally, we investigate the influence of the parameters. The crack tip speed depends on the displacement load and the fracture toughness. It increases with the displacement load and decreases with the fracture toughness (Tables 5 and 6 ). If the load displacement is too small or the fracture toughness too large, the crack does not propagate. We observe that, with about 4-8 elements in the cohesive zone, the crack tip speed is accurate to about $1 \%$. 


\begin{tabular}{|c|c|c|c|c|c|c|c|c|}
\hline$n_{\text {mesh }}$ & $\nu_{C}$ & $l_{c o h} / \Delta x$ & $l_{c r}(T)$ & $v_{c r}(T / 2, T)$ & $E_{e l}(T)$ & $E_{k i n}(T)$ & $E_{c o h}(T)$ & $E_{\text {tot }}(T)$ \\
\hline 100 & 1 & 1.963 & 0.03645 & 2282 & 1274 & 132.2 & 418.1 & 1825 \\
200 & 1 & 3.927 & 0.03844 & 2528 & 1235 & 123.3 & 454.4 & 1813 \\
400 & 1 & 7.854 & 0.03877 & 2556 & 1231 & 116.7 & 457.2 & 1805 \\
800 & 1 & 15.71 & 0.03876 & 2556 & 1231 & 113.8 & 456.5 & 1801 \\
\hline
\end{tabular}

Table 3 Mode I fracture. Numerical results. Scheme B.

\begin{tabular}{|c|c|c|c|c|c|c|c|}
\hline$p_{c} / C_{L}$ & $\nu_{C}$ & $l_{c r}(T)$ & $v_{c r}(T / 2, T)$ & $E_{e l}(T)$ & $E_{k i n}(T)$ & $E_{c o h}(T)$ & $E_{\text {tot }}(T)$ \\
\hline 10 & 0.88 & 0.03918 & 2561 & 1294 & 146.3 & 465.3 & 1905 \\
100 & 0.33 & 0.03944 & 2593 & 1229 & 149.2 & 470.3 & 1848 \\
1000 & 0.11 & 0.03942 & 2594 & 1216 & 138.8 & 468.9 & 1824 \\
\hline
\end{tabular}

Table 4 Mode I fracture. Numerical results. Fully explicit scheme with regularized cohesive zone model $\left(n_{\text {mesh }}=200\right)$.

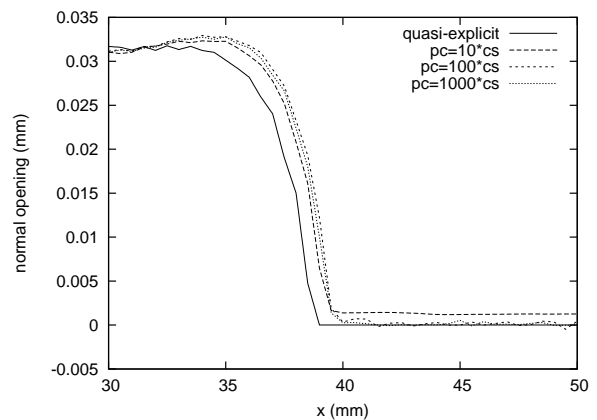

Fig. 13 Normal opening around the crack tip at time $T$. Quasi-explicit scheme B and fully explicit schemes with regularized cohesive zone model $\left(n_{m e s h}=200\right)$.

\begin{tabular}{|c|c|c|c|c|c|c|c|c|}
\hline$n_{\text {mesh }}$ & $\nu_{C}$ & $l_{\text {coh }} / \Delta x$ & $l_{c r}(T)$ & $v_{c r}(T / 2, T)$ & $E_{e l}(T)$ & $E_{k i n}(T)$ & $E_{c o h}(T)$ & $E_{\text {tot }}(T)$ \\
\hline 100 & 1 & 1.963 & 0.04196 & 2720 & 1690 & 281.8 & 512.2 & 2484 \\
200 & 1 & 3.927 & 0.04341 & 2823 & 1660 & 273.1 & 535 & 2468 \\
400 & 1 & 7.854 & 0.04373 & 2860 & 1647 & 273.1 & 538.8 & 2458 \\
800 & 1 & 15.71 & 0.04371 & 2862 & 1646 & 270.4 & 537.4 & 2454 \\
\hline \hline$n_{\text {mesh }}$ & $\nu_{C}$ & $l_{c o h} / \Delta x$ & $l_{c r}(T)$ & $v_{c r}(T / 2, T)$ & $E_{e l}(T)$ & $E_{k i n}(T)$ & $E_{c o h}(T)$ & $E_{\text {tot }}(T)$ \\
\hline 200 & 1 & 3.927 & 0.02112 & 493.7 & 1242 & 54.16 & 167.5 & 1464 \\
400 & 1 & 7.854 & 0.02209 & 618.2 & 1230 & 44.45 & 181.8 & 1457 \\
800 & 1 & 15.71 & 0.02229 & 639 & 1228 & 40.55 & 184.7 & 1453 \\
\hline
\end{tabular}

Table 5 Mode I fracture. Numerical results for different displacement loads. Scheme B. $\epsilon=$ 0.0035 (top). $\epsilon=0.0027$ (bottom).

7.2 Mode I fracture with a rate-dependent cohesive zone model

We still consider the same mode-I fracture problem except that the cohesive zone model is now a triangular rate-dependent cohesive zone model with only normal force (the viscosity parameter is denoted $\eta$ ). The notation and the parameters are the same. As expected, the introduction of a viscosity parameter slows down the crack tip (Figure 14 right, and Table 7). We observe a certain variation in the crack 


\begin{tabular}{|c|c|c|c|c|c|c|c|c|}
\hline$n_{\text {mesh }}$ & $\nu_{C}$ & $l_{\text {coh }} / \Delta x$ & $l_{c r}(T)$ & $v_{c r}(T / 2, T)$ & $E_{e l}(T)$ & $E_{k i n}(T)$ & $E_{c o h}(T)$ & $E_{\text {tot }}(T)$ \\
\hline 100 & 1 & 0.9817 & 0.04195 & 2686 & 1308 & 253 & 256 & 1817 \\
200 & 1 & 1.963 & 0.04494 & 2865 & 1266 & 266.8 & 280 & 1813 \\
400 & 1 & 3.927 & 0.04614 & 2944 & 1240 & 276.5 & 288.4 & 1805 \\
800 & 1 & 7.854 & 0.04648 & 2969 & 1230 & 281.5 & 291.7 & 1803 \\
1600 & 1 & 15.71 & 0.04654 & 2982 & 1226 & 283.6 & 292.1 & 1802 \\
\hline \hline$n_{\text {mesh }}$ & $\nu_{C}$ & $l_{\text {coh }} / \Delta x$ & $l_{c r}(T)$ & $v_{c r}(T / 2, T)$ & $E_{e l}(T)$ & $E_{k i n}(T)$ & $E_{c o h}(T)$ & $E_{\text {tot }}(T)$ \\
\hline 50 & 1 & 1.963 & 0.03326 & 1922 & 2336 & 237.2 & 722.2 & 3296 \\
100 & 1 & 3.927 & 0.03516 & 2089 & 2258 & 195.7 & 771.7 & 3225 \\
400 & 1 & 7.854 & 0.03554 & 2157 & 2247 & 168.4 & 784.4 & 3199 \\
400 & 1 & 15.71 & 0.0357 & 2168 & 2246 & 153.7 & 787 & 3187 \\
\hline
\end{tabular}

Table 6 Mode I fracture. Numerical results for different fracture toughness. Scheme B. $\sigma_{c}=$ $1.2, G_{c}=8000, \epsilon=0.004$ (top). $\sigma_{c}=1.2, G_{c}=32000, \epsilon=0.004$ (bottom).

tip speed for $\eta=0.01$ and $\eta=0.02$. Indeed, in these cases, the crack tip speed is sufficiently low so that the mechanical waves generated by the crack growth and reflected on the boundary can perturb the crack tip advance. Note that the total energy is not preserved in this problem since the rate-dependent cohesive zone model is dissipative.

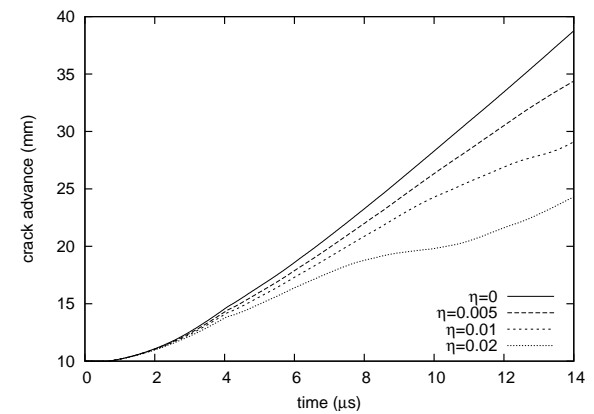

Fig. 14 Mode I fracture with a rate-dependent cohesive zone model. Crack tip advance for different viscosity parameters.

\begin{tabular}{|c|c|c|c|c|c|c|}
\hline$\eta$ & $l_{c r}(T)$ & $v_{c r}(T / 2, T)$ & $E_{e l}(T)$ & $E_{k i n}(T)$ & $E_{c o h}(T)$ & $E(T)$ \\
\hline 0 & 0.03877 & 2556 & 1231 & 116.7 & 457.2 & 1805 \\
0.005 & 0.0345 & 2083 & 1289 & 83.43 & 384.6 & 1757 \\
0.01 & 0.02926 & 1453 & 1379 & 74.61 & 299.7 & 1754 \\
0.02 & 0.0244 & 953 & 1471 & 60.24 & 220 & 1751 \\
\hline
\end{tabular}

Table 7 Mode I fracture with a rate-dependent cohesive zone model. Numerical results for different viscosity parameters (Scheme B, $n_{\text {mesh }}=400, \nu_{C}=1$ ).

The rate dependence of the cohesive zone model introduces a new time scale in the problem, linked to the opening rate. This time scale is smaller than the time scale linked to the crack tip speed and requires therefore smaller time steps to be 
resolved. For instance, we observe small oscillations on the crack tip advance when a too coarse discretization is used.

\subsection{Mode II fracture}

In this section, we consider an example of mode-II fracture (Figure 15). The domain is the rectangle $\Omega=\left(0, L_{x}\right) \times\left(-L_{y}, L_{y}\right)$. The fracture interface is the line $\Gamma=\left(0, L_{x}\right) \times\{0\}$. The cohesive zone model is a reversible uncoupled triangular model with a fracture toughness $G_{c}$ and a critical stress $\sigma_{c}$. A constant shear force is prescribed at the sides of the interface $\left(f_{s} e_{x}\right.$ is enforced at the top side and $-f_{s} e_{x}$ at the bottom side, with $\left.f_{s}=\sigma_{c} / 2\right)$. At the interface, the normal opening is enforced to zero, so that the fracture is in pure mode II. At the initial time, a precrack of length $L_{\text {pre }}$ is inserted at mid-height on the left edge.

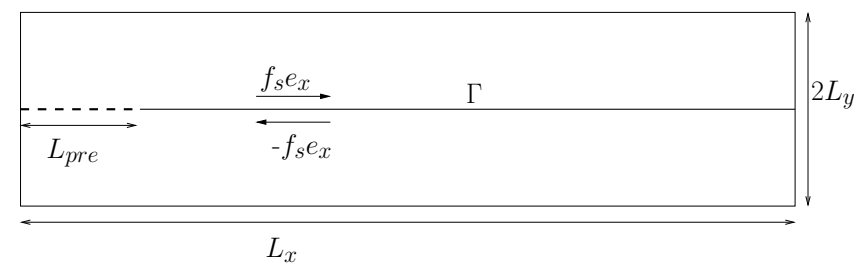

Fig. 15 Mode II fracture. Geometric setup.

Owing to antisymmetry, numerical simulations are performed only on the upper half part of the domain. This half-domain is uniformly meshed with half-square triangles. The parameters and the notation are the same as in the previous problems, except for the obvious change in the definition of the cohesive energy as

$$
E_{c o h}(t):=\sum_{i \in \mathcal{N}^{c}} \beta_{i} \Psi_{2}\left(\llbracket U_{h}(t) \rrbracket_{i, 2}\right)
$$

The sum of the kinetic, elastic, and cohesive energy is expected to be equal to the work of the shear force (denoted $W_{\text {ext }}$ ).

This simulation of mode-II fracture exhibits the same kind of behavior as the simulation of mode-I fracture. The precrack opens by sliding, and there is a stress concentration around the precrack tip; as soon as this stress reaches the cohesive critical stress, the crack starts growing; the crack tip speed reaches quickly a limit value, slightly lower than the dilatational wave speed. The deformation and stress fields at time $T$ are represented in Figure 16.

The observed stability condition is $\nu_{C} \lesssim 0.98$. With a mesh resolving the estimated cohesive zone length and $\nu_{C}=0.98$, the numerical results obtained with Scheme B are very accurate (Table 8) with a relative error on the energy balance less than $0.1 \%$ even for $n_{\text {mesh }}=100$, while two significant digits for crack length and average speed are reached for $n_{m e s h}=400$. 


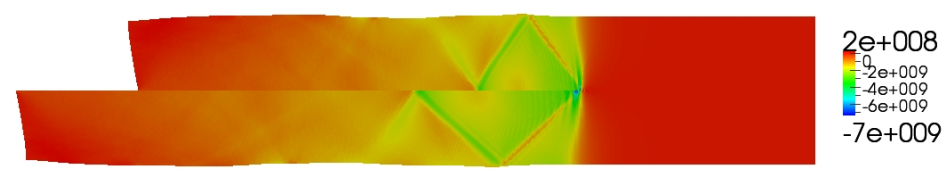

Fig. 16 Mode II fracture. Deformed configuration (enlarged 20 times) and stress $\sigma_{x x}$ at time $T$. Scheme B $\left(n_{\text {mesh }}=800, \nu_{C}=0.98\right)$.

\begin{tabular}{|c|c|c|c|c|c|c|c|c|}
\hline$n_{\text {mesh }}$ & $\nu_{C}$ & $l_{\text {coh }} / \Delta x$ & $l_{c r}(T)$ & $v_{c r}(T / 2, T)$ & $E_{e l}(T)$ & $E_{k i n}(T)$ & $E_{\text {coh }}(T)$ & $E_{\text {tot }}(T)-W_{\text {ext }}(T)$ \\
\hline 100 & 0.98 & 1.964 & 0.06542 & 4721 & 6637 & 13383 & 881.9 & -18.6 \\
200 & 0.98 & 3.93 & 0.06687 & 4804 & 6686 & 14267 & 907.3 & -8.8 \\
400 & 0.98 & 7.86 & 0.06765 & 4852 & 6714 & 14675 & 920.7 & -4.8 \\
800 & 0.98 & 15.71 & 0.06789 & 4878 & 6706 & 14768 & 923.8 & -2.9 \\
\hline
\end{tabular}

Table 8 Mode II fracture. Numerical results. Scheme B.

\subsection{Mode I fracture with contact}

This last problem involves mode-I fracture and contact (Figure 17). The domain is the rectangle $\Omega=\left(0, L_{x}\right) \times\left(-L_{y}, L_{y}\right)$. The fracture interface is the line $\Gamma=$ $\left(0, L_{x}\right) \times\{0\}$. There is a precrack of length $L_{\text {pre }}$ at mid-height on the left edge. The cohesive zone model is an irreversible triangular model with linear unloading. A constant displacement load is prescribed at the upper left edge and lower left edge $\left(u_{y}=u_{d}\right.$ at the upper edge and $u_{y}=-u_{d}$ at the lower edge, with $\left.u_{d}=\epsilon L_{y}\right)$. The initial state corresponds to the static equilibrium with Neumann condition on the precrack and Dirichlet condition on the rest of $\Gamma$ (Figure 19, top).

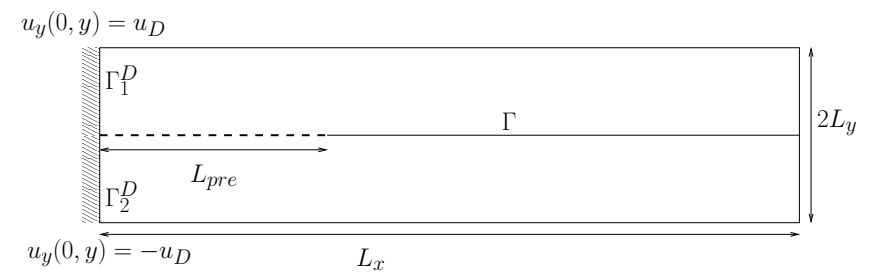

Fig. 17 Mode I fracture with contact. Geometric setup.

Owing to symmetry, numerical simulations are performed only on the upper half part of the domain. We use an unstructured triangular mesh. The parameters are the same as in the previous problems except the precrack length $\left(L_{\text {pre }}=40\right.$ $\mathrm{mm})$, the displacement load $(\epsilon=0.09)$, and the simulation time $(T=100 \mu \mathrm{s})$. The notation is essentially the same. We change the definition of the cohesive energy and define a dissipated cohesive energy as

$$
E_{c o h}(t):=\sum_{i \in \mathcal{N}^{c}} \beta_{i} \Psi_{1}^{i r r}\left(\Delta(t)_{h, i, 1}, \llbracket U_{h}(t) \rrbracket_{i, 1}\right)
$$




$$
\tilde{E}_{c o h}(t):=\sum_{i \in \mathcal{N}^{c}} \beta_{i} \tilde{\Psi}_{1}^{i r r}\left(\Delta(t)_{h, i, 1}\right) .
$$

The total energy is defined as the sum of the elastic, kinetic, cohesive, and dissipated cohesive energy. In the continuous problem, the total energy is expected to be constant.

For this problem, we observe the following behavior. At the initial time, there is a stress concentration around the precrack tip so the crack starts immediately growing (not uniformly). After a while, the crack stops growing and contact phenomena occur near the crack tip. This behavior is illustrated in Figure 18 (crack tip advance and energy evolution) and Figure 19 (deformation and stress fields at time $T / 2$ ).
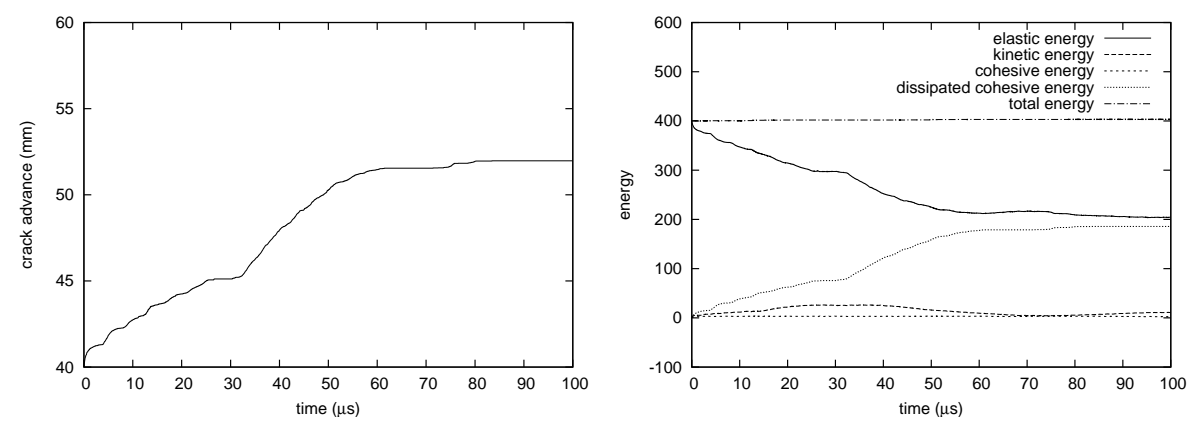

Fig. 18 Mode I fracture with contact. Crack tip advance (left) and energy evolution (right). Scheme B $\left(n_{\text {mesh }}=800, \nu_{C}=0.8\right)$.

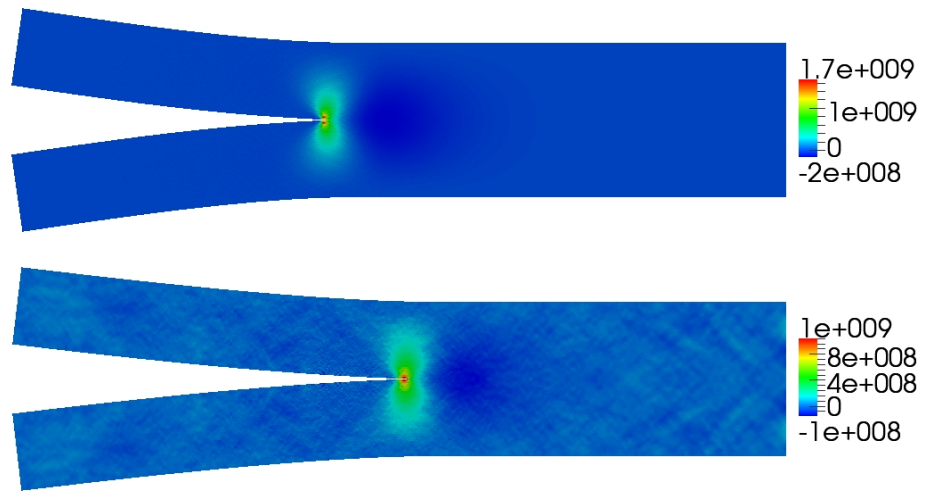

Fig. 19 Mode I fracture with contact. Deformed configuration (enlarged 5 times) and stress $\sigma_{x x}$ at initial time (top) and time $T / 2$ (bottom). Scheme B $\left(n_{m e s h}=800, \nu_{C}=0.8\right)$.

The observed stability condition is $\nu_{C} \lesssim 0.8$. With a mesh resolving the estimated cohesive zone length and $\nu_{C}=0.8$, the numerical results obtained with 
Scheme B are again very accurate (Table 9) with typically two significant digits reached for $n_{m e s h}=400$.

\begin{tabular}{|c|c|c|c|c|c|c|c|c|}
\hline$n_{\text {mesh }}$ & $\nu_{C}$ & $l_{c o h} / \Delta x$ & $l_{c r}(T)$ & $E_{e l}(T)$ & $E_{k i n}(T)$ & $E_{c o h}(T)$ & $\tilde{E}_{c o h}(T)$ & $E_{\text {tot }}(T)$ \\
\hline 100 & 0.8 & 1.963 & 0.05364 & 199.9 & 11 & 1.66 & 208.7 & 421.3 \\
200 & 0.8 & 3.93 & 0.05257 & 202 & 13.7 & 2.073 & 194.8 & 412.5 \\
400 & 0.8 & 7.86 & 0.05197 & 204.6 & 10.82 & 1.988 & 185.8 & 403.2 \\
800 & 0.8 & 15.71 & 0.0519 & 205.1 & 10.67 & 1.86 & 184.9 & 402.5 \\
\hline
\end{tabular}

Table 9 Mode I fracture with contact. Numerical results. Scheme B.

\section{Conclusions}

We have proposed and analyzed two quasi-explicit time-integration schemes for solving dynamic fracture problems with set-valued cohesive zone models. These schemes combine a central difference time-integration scheme and a partially implicit and lumped treatment of the cohesive forces. The numerical results presented in Section 7 on mode-I and mode-II fracture problems on various two-dimensional settings indicate that the present methodology provides a robust way of treating the set-valued cohesive zone model while keeping a moderate computational cost. Future work should include testing these schemes on three-dimensional configurations.

\section{References}

1. Barenblatt, G.: The formation of brittle cracks during brittle fracture. General ideas and hypotheses. Axially-symmetric cracks. Appl. Math. Mech 23, 1273-1282 (1959)

2. Barenblatt, G.: The mathematical theory of equilibrium cracks in brittle fracture. Advances in applied mechanics $7(2), 55-129$ (1962)

3. Broberg, K.: Cracks and fracture. Academic Pr (1999)

4. Cagnetti, F.: A vanishing viscosity approach to fracture growth in a cohesive zone model with prescribed crack path. Math. Models Methods Appl. Sci. 18(7), 1027-1071 (2008)

5. Camacho, G.T., Ortiz, M.: Computational modelling of impact damage in brittle materials. International Journal of Solids and Structures 33(20-22), $2899-2938$ (1996)

6. Cohen, G., Joly, P., Roberts, J., Tordjman, N.: Higher order triangular finite elements with mass lumping for the wave equation. SIAM Journal on Numerical Analysis 38(6), 2047-2078 (2001)

7. Corigliano, A., Ricci, M.: Rate-dependent interface models: formulation and numerical applications. International Journal of Solids and Structures 38(4), $547-576$ (2001)

8. Dal Maso, G., Zanini, C.: Quasi-static crack growth for a cohesive zone model with prescribed crack path. Proc. Roy. Soc. Edinburgh Sect. A 137(2), 253-279 (2007)

9. Doyen, D., Ern, A.: Convergence of a space semi-discrete modified mass method for the dynamic Signorini problem. Commun. Math. Sci. 7(4), 1063-1072 (2009)

10. Doyen, D., Ern, A., Piperno, S.: A three-field augmented Lagrangian formulation of unilateral contact problems with cohesive forces. ESAIM, Math. Model. Numer. Anal. 44(2), 323-346 (2010)

11. Dugdale, D.S.: Yielding of steel sheets containing slits. Journal of the Mechanics and Physics of Solids 8(2), $100-104$ (1960)

12. Falk, M., Needleman, A., Rice, J.: A critical evaluation of cohesive zone models of dynamic fractur. Le Journal de Physique IV 11(PR5) (2001) 
13. Freund, L.B.: Dynamic fracture mechanics. Cambridge Monographs on Mechanics and Applied Mathematics. Cambridge University Press, Cambridge (1990)

14. Hecht, F., Pironneau, O.: Freefem++. Www.freefem.org

15. Hughes, T.J.R.: The finite element method. Prentice Hall Inc., Englewood Cliffs, NJ (1987)

16. Lorentz, E.: A mixed interface finite element for cohesive zone models. Computer Methods in Applied Mechanics and Engineering 198(2), 302 - 317 (2008)

17. Marigo, J.J., Truskinovsky, L.: Initiation and propagation of fracture in the models of Griffith and Barenblatt. Contin. Mech. Thermodyn. 16(4), 391-409 (2004)

18. Paggi, M., Carpinteri, A., Zavarise, G.: A unified interface constitutive law for the study of fracture and contact problems in heterogeneous materials. In: P. Wriggers, U. Nackenhorst (eds.) Analysis and Simulation of Contact Problems, Lecture Notes in Applied and Computational Mechanics, vol. 27, pp. 297-304. Springer Berlin / Heidelberg (2006)

19. Pandolfi, A., Guduru, P.R., Ortiz, M., Rosakis, A.J.: Three dimensional cohesive-element analysis and experiments of dynamic fracture in c300 steel. International Journal of Solids and Structures 37(27), 3733 - 3760 (2000)

20. Ravi-Chandar, K., Knauss, W.: An experimental investigation into dynamic fracture: III. On steady-state crack propagation and crack branching. International Journal of Fracture 26(2), 141-154 (1984)

21. Samudrala, O., Huang, Y., Rosakis, A.J.: Subsonic and intersonic mode ii crack propagation with a rate-dependent cohesive zone. Journal of the Mechanics and Physics of Solids $\mathbf{5 0}(6), 1231-1268(2002)$

22. Sharon, E., Gross, S., Fineberg, J.: Energy dissipation in dynamic fracture. Physical review letters 76(12), 2117-2120 (1996)

23. Talon, C., Curnier, A.: A model of adhesion coupled to contact and friction. European Journal of Mechanics - A/Solids 22(4), 545 - 565 (2003)

24. Turon, A., Dvila, C., Camanho, P., Costa, J.: An engineering solution for mesh size effects in the simulation of delamination using cohesive zone models. Engineering Fracture Mechanics 74(10), 1665 - 1682 (2007)

25. Willis, J.R.: A comparison of the fracture criteria of griffith and barenblatt. Journal of the Mechanics and Physics of Solids 15(3), 151 - 162 (1967)

26. Xu, X.P., Needleman, A.: Numerical simulations of fast crack growth in brittle solids. Journal of the Mechanics and Physics of Solids 42(9), 1397 - 1434 (1994)

27. Zhou, F., Molinari, J.F., Shioya, T.: A rate-dependent cohesive model for simulating dynamic crack propagation in brittle materials. Engineering Fracture Mechanics 72(9), 1383 $-1410(2005)$ 